\title{
Cost-effective epigenetic age profiling in shallow methylation sequencing data
}

\author{
Alexandre Trapp \& Vadim N. Gladyshev*
}

Division of Genetics, Department of Medicine, Brigham and Women's Hospital, Harvard Medical School, Boston, MA 02115, USA

* Corresponding author. e-mail: vgladyshev@,rics.bwh.harvard.edu 


\begin{abstract}
There is a critical need for robust, high-throughput assays of biological aging trajectories. Among various approaches, epigenetic aging clocks emerged as reliable molecular trackers of the aging process. However, current methods for epigenetic age profiling are inherently costly and lack throughput. Here, we leverage the scAge framework for accurate prediction of biological age from very few bisulfite sequencing reads in bulk samples, thereby enabling drastic (100-1,000-fold) reduction in sequencing costs per sample. Our approach permits age assessment based on distinct assortments of $\mathrm{CpG}$ sites in different samples, without the need for targeted site enrichment or specialized reagents. We demonstrate the efficacy of this method to quantify the age of mouse blood samples across independent cohorts, identify the effect of calorie restriction as an attenuator of the aging process, and discern rejuvenation upon cellular reprogramming. We propose that this framework may be used for epigenetic age prediction in extremely high-throughput applications, enabling robust, large-scale and inexpensive interventions testing and age profiling.
\end{abstract}




\section{Introduction}

Mammalian aging is characterized by profound changes in the epigenomic landscape, particularly in the DNA methylome ${ }^{1,2}$. Cytosine methylation fractions at $\mathrm{CpG}$ dinucleotides can be detected using next-generation sequencing approaches — both genome-wide and targeted - as well as microarray technologies ${ }^{3-7}$. These methods permit high-resolution characterization of methylation patterns in bulk tissue samples, and more recently in single cells ${ }^{8,9}$. While methylation plays an active role in a variety of downstream biological processes, its relationship with aging has been of special interest in recent years. In fact, methylation dynamics have been integrated with artificial intelligence techniques (including both shallow and deep machine learning approaches) to enable accurate age estimation based on DNA methylation profiles in humans, mice, and other mammalian organisms ${ }^{7,10-15}$.

Epigenetic aging clocks, tools that quantitatively leverage coordinated age-associated changes in the DNA methylome, are believed to reflect an inherent measure of biological age in their predictions ${ }^{16}$. While chronological age is fixed based upon one's date of birth, biological age is conceived of as an integrative and fluid metric that accounts for intrinsic and environmental factors, which are ultimately reflected in the dynamic DNA methylome. Epigenetic age, specifically measured by tracking concerted changes in particular $\mathrm{CpG}$ methylation patterns, has hence emerged as one of the most promising "biomarkers" of the aging process, and has been used as a platform to evaluate the effect of longevity and rejuvenation interventions ${ }^{17,18}$. This line of inquiry is especially promising, given that epigenetic aging dynamics have recently been shown to exhibit remarkable conservation across mammalian species ${ }^{19-22}$.

However, current methods for epigenetic age profiling are much too costly and lowthroughput. In humans, the most common approach for partial methylome analysis remains the 
various suite of methylation arrays produced by Illumina (including the depreciated $27 \mathrm{~K} \& 450 \mathrm{~K}$ chips, and the more recent EPIC Infinium chip) ${ }^{7,23}$. These microarrays leverage bisulfite conversion and fluorescent probes that ultimately enable reproducible assessment of the fraction of methylation in a bulk sample (known as the beta values) at several thousand $\mathrm{CpGs}^{24}$. In mice, the lack of methylation arrays (until recently ${ }^{19,25}$ ) has restricted profiling to reduced representation bisulfite sequencing (RRBS), a method that involves restriction-enzyme-mediated CpG island enrichment, bisulfite conversion, and short-read next-generation sequencing ${ }^{11,18}$. With both of these approaches, there is a requirement for considerable amounts of input DNA, in part due to harsh chemical treatment by sodium bisulfite during library preparation. Additionally, the costs (either of the microarray or the sequencing runs) can be prohibitively high for routine applications. This poses a profound limitation for large-scale efforts to profile biological age in populations or cohorts, particularly in regard to throughput, labor, time and cost.

To address these issues, we leveraged our recently developed $s c$ Age framework ${ }^{26}$ to enable robust profiling of epigenetic age in low-pass bulk bisulfite sequencing data. By downsampling deeply-sequenced RRBS data, we arrived at a data type very similar to single cells: sparse, mostly binary methylation values. The use of few sequencing reads further results in variable coverage of cytosines, despite the use of enriched methylation protocols. To address this, our method makes use of different $\mathrm{CpG}$ sites in each sample for epigenetic age predictions, circumventing the need for a set of CpGs to be consistently covered across many samples. Here, we introduce our framework as a tool for inexpensive and high-throughput epigenetic age predictions, particularly well-suited for large-scale studies. 


\section{Results}

\section{Simulating low-pass bisulfite sequencing data}

To develop a platform for epigenetic age profiling from low-coverage bisulfite sequencing data, we first designed a simulation pipeline that enables the creation of reduced methylation profiles from randomly downsampled sequencing reads (Fig. 1a). We utilized existing RRBS data from mouse aging studies ${ }^{10,27}$, wherein individual libraries were deeply sequenced at more than $10^{7}$ reads per sample. Next, we devised a random downsampling algorithm that takes as input individual reads and outputs a desired number of subsampled reads. Practically, we downsampled bulk data to $10^{2}, 10^{3}, 10^{4}$ and $10^{5}$ reads, enabling comprehensive assessment of different low-pass bisulfite sequencing outputs.

Since bulk RRBS protocols commonly enable readout at a few million CpGs per sample, downsampling to a low number of reads or performing low-pass sequencing limits the applicability of conventional epigenetic clock approaches. These methods traditionally rely on training machine learning models - usually based on elastic-net regularization-which effectively select informative CpGs and assign them weights in a resulting linear model. Subsequently, new methylation values, weighted by the model and often adjusted by an intercept, can be directly used for epigenetic age predictions ${ }^{7,10,12,27-29}$. However, the algorithms driving these models require that many CpGs are covered at high depth consistently across many samples, which can presently only be accomplished by deep sequencing or methylation array technologies. Additionally, once a set of $\mathrm{CpG}$ sites is chosen and given weights in the model, the same $\mathrm{CpG}$ sites must all be present in any future testing dataset in order to obtain the most accurate predictions. This can be circumvented to some degree with imputation approaches ${ }^{12,27,30,31}$, but an excessive number of missing values tends to greatly reduce the absolute accuracy of current clocks ${ }^{32}$. While intersecting bulk genomic 
RRBS data produces large feature tables (on the order of $10^{5}-10^{6} \mathrm{CpGs}$ ) amenable to machine learning approaches, low-pass sequencing profiles have minimal common sites, precluding the use of conventional methods (Fig. 1b). Indeed, feature tables constructed from aggregated shallow data are a stark contrast to those built from deep data, specifically differing in their primary data type and their sparsity (Fig. 1c).

To address this key challenge, we leveraged the scAge framework, which is particularly amenable to sparsely covered methylation data with distinct sets of $\mathrm{CpG}$ sites available in each individual sample. Briefly, the framework first involves training CpG-specific regression models predicting methylation level from chronological age. Next, our platform employs a ranked intersection algorithm: only $\mathrm{CpG}$ sites covered both in a particular sample of interest and the reference data are retained, and these are subsequently ranked and filtered to output only highly age-associated common $\mathrm{CpG}$ sites (Fig. 1d, e). The algorithm then cycles through each $\mathrm{CpG}$ site in the resulting profile, and measures the distance between the observed methylation value and the estimate from the linear model at a particular age. We propose to interpret this distance metric as a probability, meaning that ages with smaller absolute distance between the observed value and the model prediction are more representative of the true biological age of that particular sample (Fig. 1f). Finally, we amass several hundred/thousand CpG-specific probabilities into a comprehensive likelihood profile, which enables efficient epigenetic age profiling using sparse methylation data (Fig. 1g).

The scAge framework was initially developed to profile epigenetic age using highresolution single-cell methylation data ${ }^{26}$. Here, we adjusted the original algorithm to conform to an additional parameter resulting from its application to bulk samples: meaningful non-binary methylation. In single cells, the binary nature of DNA methylation data is mostly reflective of 
inherent biology in major cell types. However, bulk samples (even downsampled ones) may exhibit meaningful non-binary methylation, given that sequencing reads come from many different cells in the sample. In line with this notion, we modified our algorithm to accommodate for nonbinary methylation data. Altogether, we developed a pipeline that can take as input real or simulated low-pass methylation data for efficient epigenetic age predictions.

As reference data for scAge, we used normal, standard ad libitum C57BL/6J mouse blood samples from the Petkovich et al. ${ }^{10}(\mathrm{n}=153)$ and Thompson et al. ${ }^{27}(\mathrm{n}=50)$ studies. Samples ranged in age from 1 month to 35 months in the Petkovich et al. data, and 2 to 21 months in the Thompson et al. data. We selected highly covered (depth $\geq 5 \mathrm{x}$ ) CpG sites in each sample and constructed a large feature table for all the samples in a dataset across all identified CpGs. Upon additional filtering, this generated two independent reference sets: 1.2 million CpG-specific linear models in the Thompson et al. ${ }^{27}$ data and 1.9 million CpG-specific linear models in the Petkovich et al. ${ }^{10}$ data. Of note, several differences in data processing, experimental protocols, and the number of samples in each dataset likely account for the variability in the number of trainable $\mathrm{CpG}$ linear models we obtained for each dataset.

\section{scAge tracks the aging process in blood based on low-pass sequencing data}

We first trialed our approach on downsampled data from the Thompson et al. ${ }^{27}$ study (Fig. 2a). We selected $10^{2}, 10^{3}, 10^{4}$ and $10^{5}$ reads, each across 5 random states; in effect, this enabled randomization of the particular set of CpGs in each subsample, and mirrored sequencing small random aliquots of libraries. For each of these random, downsampled $\mathrm{CpG}$ methylation matrices, we applied our modified scAge framework trained on all samples from either the Petkovich et al. ${ }^{10}$ or Thompson et al. ${ }^{27}$ studies. We benchmarked the framework and its predictions across several 
read number parameters and likelihood profile sizes, using anywhere from 50 to $10^{4}$ top ageassociated CpGs per sample for age computations.

Excitingly, we observed robust performance of our epigenetic age profiling approach in these data, with the results varying based on the number of total CpGs in the downsampled data, the number of age-associated CpGs included in the likelihood profile, and the particular reference models employed. Pearson correlation coefficients $(r)$ generally increased in magnitude and significance as more reads were subsampled, with some variation based on the size of the likelihood profile (Fig. 2b, c). Mean and median absolute errors decreased using both models as the number of reads was increased, again with some minor variation depending on profile sizes (Fig. 2d, e). Using these benchmarking results, we conducted further analysis on the best performing set of parameters according to both training datasets $(100,000$ reads with the top 1,000 age-associated CpGs included in the likelihood computation profile). When Thompson et al. ${ }^{27}$ linear models were used as reference, correlation coefficients ranged from 0.93-0.96 depending on the random seed (Extended Data Fig. 1). Application of the independent Petkovich et al. ${ }^{10}$ models showed slightly decreased predictive accuracy, with Pearson correlations ranging from 0.81-0.85. However, median errors were remarkably low with both training datasets, ranging from 1.81-2.35 months with Thompson et al. references models and 2.56-3.48 months with Petkovich et al. reference models. Interestingly, predictive metrics were almost equally robust when only 10,000 reads per sample were assayed (Fig. 2b-e). This suggests that integration of the scAge framework and low-pass sequencing - producing a relatively small number of reads - may be sufficient to accurately profile biological age.

Next, we applied the same methodology on downsampled data from the Petkovich et al. ${ }^{10}$ study, which included 153 blood samples from standard ad libitum male C57BL/6J mice aged 
1-35 months (Fig. 3a). Again, we observed strong performance across both reference models on these data, with similar evaluation trends as what we observed upon application to downsampled Thompson et al. ${ }^{27}$ data (Fig. 3b-e). Indeed, increasing the number of reads was associated with improved prediction accuracy across all metrics, and results varied based on the specific number of CpGs leveraged by scAge. When selecting the most accurate parameters based on benchmarking (100,000 reads per sample with the top 500 age-associated CpGs included in the likelihood profile), Pearson $r$ coefficients ranged from 0.88-0.9, and median absolute errors ranged from 2.9 to 3.2 months using the Petkovich et al. reference models. However, the Thompson et al. models were noticeably less accurate, particularly in regard to absolute error $(r=0.71-0.74$, MedAE $=7.0$ 7.6m) (Extended Data Fig. 2). We hypothesize this may be largely due to differential processing of the Thompson et al. ${ }^{27}$ methylation data as compared to the Petkovich et al. ${ }^{10}$ data, and may also be reflective of batch effects. Indeed, we observed only a moderate positive association between CpG-specific Pearson correlations $(r=0.43)$ or linear regression coefficients $(r=0.54)$ with age among the Petkovich et al. and Thompson et al. reference datasets (Extended Data Fig. 3).

\section{Prediction consistency suggests reproducibility in low-pass epigenetic age profiling}

In order to effectively simulate different sequencing runs of the same sample, we applied a random downsampling approach that returned distinct sets of $\mathrm{CpGs}$ depending on the chosen seed. This procedure can be thought of as computationally analogous to picking a random aliquot of a library for sequencing, and is particularly suited for tracking the consistency of predictions from different subsamples (Fig. 4a). Excitingly, the best performing predictive tests (those with 10,000-100,000 reads) showed really strong inter-subsample prediction consistency. Indeed, both Thompson et al. and Petkovich et al. models had inter-subsample Pearson correlation coefficients greater than 0.9 
when testing on downsampled Thompson et al. ${ }^{27}$ data (Fig. 4b). The Petkovich et al. ${ }^{10}$ models similarly showed robust inter-subsample prediction correlations when applied to Petkovich et al. ${ }^{10}$ data, while the Thompson et al. ${ }^{27}$ models applied to this same dataset had slightly weaker correlations (Fig. 4c). Overall, these results suggest that our approach is able to generate reproducible predictions from different small assortments of covered $\mathrm{CpGs}$, reinforcing the putative value of our framework for handling shallow data.

\section{Validation of attenuated aging with caloric restriction using low-pass epigenetic age profiling}

Next, we were interested if our low-pass scAge framework could distinguish the effect of caloric restriction as a longevity intervention. Caloric restriction was previously found to attenuate biological aging as measured in blood ${ }^{10}$ and liver ${ }^{13}$ samples, and is commonly considered one of the gold-standard lifespan-extending interventions currently in use in model systems ${ }^{33,34}$. To test the effectiveness of our approach, we analyzed 20 blood samples from male calorie-restricted C57BL/6J mice with chronological ages ranging from 10 to 27 months (Fig. 5a). Of note, these samples were completely independent from the Petkovich et al. linear model reference set used for scAge predictions.

Remarkably, by using these standard-diet Petkovich et al. ${ }^{10}$ models integrated into scAge, we were able to reliably detect a significant decrease in delta age (i.e., the difference between epigenetic and chronological age) for calorically restricted mice compared to control when either $10^{4}$ or $10^{5}$ reads per sample were utilized (Fig. 5b). In the best performing test case based on benchmarking $(100,000$ reads per sample with the top 2,500 CpGs included in the likelihood profile), delta age in calorically restricted mice was significantly $(p<0.0001)$ lower than in mice fed ad libitum across every random subsample (Extended Data Fig. 4). Moreover, linear 
regression analyses revealed a significantly attenuated epigenetic aging trajectory for calorierestricted mice (Fig. 5c). This suggests that our method is capable of reproducibly discerning the effect of potent longevity interventions, despite having relatively few sequencing reads. However, it is important to note that application of the Thompson et al. models did not reveal a significant effect. Again, we hypothesize this is largely a manifestation of impaired accuracy of the Thompson et al. models on the Petkovich et al. data as a whole (Fig. 3b-e), which may be caused by processing incompatibilities, protocol differences, or other significant batch effects (Extended

\section{Data Fig. 3).}

\section{Low-pass scAge identifies age reversal effect upon iPSC reprogramming}

Given the rising interest in cellular reprogramming approaches for rejuvenation research, we were interested if our low-pass approach, combined with the scAge framework, could identify a significant epigenetic age decrease resulting from iPSC reprogramming ${ }^{18,35,36}$. We applied our method, trained on blood methylation data, to renal and lung fibroblasts and corresponding iPSC lines derived from these tissues. Despite a rather small sample size ( $n=3$ cell lines per group), we observed that predicted epigenetic ages based on Petkovich et al. blood regression models were significantly lower for iPSCs than for fibroblasts across all random subsampling states tested when using 100,000 reads (Fig. 6). When selecting the best test case based on benchmarking (100,000 reads per sample with the top $500 \mathrm{CpGs}$ included in the likelihood profile), we witnessed significant decreases in epigenetic age across almost all random subsamples using both the Petkovich et al. and Thompson et al. reference models, in both kidney-derived and lung-derived iPSCs (Extended Data Fig. 5, 6). Absolute age predictions had improved accuracy under 
Petkovich et al. models, with iPSC samples displaying epigenetic ages near 0 , validating results from earlier deep sequencing, elastic-net based approaches ${ }^{7,32}$.

Together, these data suggest that epigenetic age reversal by Yamanaka factor-based induction of pluripotency (conventional iPSC generation) can be assayed using low-pass approaches in combination with our clock framework, inviting further applications for the robust, inexpensive, and high-throughput evaluation of putative rejuvenation interventions.

\section{Low-coverage profiling uncovers the effect of genetic manipulations}

In addition to dietary and reprogramming-based interventions, we were interested in testing if the integration of low-pass sequencing approaches with our scAge framework enables assessment of attenuated biological aging due to commonly studied genetic alterations. Some genetic manipulations, notably growth hormone receptor knockouts (GHRKO) and Pit1 loss-of-function mutations (Snell dwarf), have previously shown lifespan-extending effects as well as decreased biological age when assessed by a blood epigenetic clock $^{10,37,38}$.

We tested our approach on young GHRKO, Snell dwarf, and control mice (all independent from the Petkovich et al. reference set), and observed a trend towards age reduction, particularly when employing the Petkovich et al. models in samples with 100,000 reads and several thousand CpGs included in likelihood profiles (Fig. 7). The Thompson et al. models showed some nominal significance on GHRKO mice when 100,000 reads were downsampled, but this was not always consistent across random states. By selecting the best performing model based on benchmarking (100,000 reads per sample with the top $10,000 \mathrm{CpGs}$ in each likelihood profile), we observed significant decreases in delta age for GHRKO mice compared to control mice across both reference sets, while the attenuated aging phenotype in Snell dwarf mice could only be significantly picked 
up by scAge running on the Petkovich et al. ${ }^{10}$ reference models (Extended Data Fig. 7, 8). This is not entirely unsurprising, as previous deep sequencing elastic-net clocks have also failed to pick up statistically significant differences in Snell dwarf models ${ }^{11}$. Interestingly, delta ages obtained with Thompson et al.-based scAge predictions were not centered around 0 (as is expected and was the case with Petkovich et al. models), reinforcing the likely presence of batch effects.

Altogether, we show that our new approach may discern the attenuated epigenetic aging effect brought on by genetic manipulations. Hence, low-pass sequencing combined with our computational age profiling platform opens avenues for large-scale genetic screening applications.

\section{Discussion}

We report here the development and application of the epigenetic clock framework scAge to shallow bulk RRBS sequencing data, revealing robust performance at age prediction across independent murine blood datasets (Fig. 1-3). We randomly downsampled existing deep RRBS data, followed by epigenetic age profiling leveraging the scAge approach. As expected, predictions were stronger when the linear model reference set used came from the same study as the downsampled data, but independent reference datasets still performed well, suggesting that our approach should be generalizable to new collections of data.

Interestingly, we show that scAge combined with low-pass sequencing is amenable to reproducible profiling of biological age (Fig. 4). Our approach also enables tracking the attenuated biological aging effect brought on by calorie restriction (Fig. 5), as well as age reversal by induction of pluripotency through the Yamanaka factors in fibroblast lines from two tissues (Fig. 6). However, we note that we observe the most significant results for these interventions in the case where predictions are based on training data sourced from the same dataset as the 
downsampled data. This suggests the importance of acknowledging and accounting for batch effects in these analyses (Extended Data Fig. 3). Generation of additional bulk RRBS datasets amenable for training may shed more light on this phenomenon, and potentially enable universal application of our framework to other datasets. Additionally, we show that some genetic interventions - namely GHRKO and Snell dwarf mouse models-reduce biological age as predicted by scAge, but only under a more restrictive set of parameters and higher read counts (Fig. 7). Since the effect of caloric restriction can be picked up with high significance, we hypothesize that these slightly weaker results in genetic models may arise from application of a framework trained on C57BL/6J mice to different mouse strains; indeed, Snell dwarf mice and controls were of a (DW/J x C3H/HEJ)/F2 background, and GRHKO mice and controls were of a (C57BL/6J x BALB/cByJ)/F2 background.

A major advantage of our approach is the cost of sequencing. Compared to a typical RRBS protocol that yields $>10^{7}$ reads per sample, our method at present requires only $10^{4}-10^{5}$ reads, representing a 100-1,000-fold reduction in sequencing costs with appropriate multiplexing. An additional advantage of our method over those currently available is the direct use of isolated DNA samples for bisulfite sequencing, without the need to specifically enrich $\mathrm{CpG}$ sites of interest. This makes our method easily applicable to diverse data and sample sets, and does not require special expertise or reagents that are otherwise needed with methods relying on particular sets of predefined CpG sites.

While the framework we present here shows promise at accurate profiling of epigenetic age from low-pass sequencing data, there are a number of potential limitations that need to be acknowledged. First, while the approach employed in our study enables benchmarking and comparative analyses, it is not identical to high-throughput low-pass data, when more samples are 
multiplexed and sequenced in real-time. Additionally, our current method has so far only been applied in mice, although we suppose that identical or similar methods should be functional for low-cost epigenetic age profiling in human samples, as well as in other mammalian species. Our scAge approach also currently generalizes all methylation-age relationships as linear, which is likely not the most optimal mathematical reflection for the diverse set of age-related behaviors of individual CpGs. Lastly, while the use of different cytosines for age predictions in each sample enables de novo assessment of epigenetic aging (which could not otherwise be performed with conventional approaches), this reliance on diverse sets of $\mathrm{CpGs}$ may concurrently reduce the precision of predictions.

Overall, we leveraged our scAge framework for epigenetic age profiling in low-coverage bulk RRBS data. We assessed shallow data across a variety of read sets ranging from $10^{2}$ to $10^{5}$, revealing robust performance of our platform specifically when 10,000-100,000 reads are available per sample. We show that parameters of scAge have some impact on prediction metrics, although comparatively less than the number of reads sampled. Excitingly, we find that the scAge approach combined with shallow sequencing data enables prediction of biological age from as a little as 10,000 reads in standard blood samples of C57BL/6J mice across two independent datasets. Additionally, we report that our method may be amenable to identify the effects of some longevity and rejuvenation interventions — such as calorie restriction or iPSC reprogramming — on biological aging trajectories. This suggests our approach may be particularly useful to validate and identify new longevity interventions.

While epigenetic age profiling is becoming more mainstream, current methods remain prohibitively expensive for routine epigenetic testing or for large-scale assessment of biological age in human cohorts or in model systems. Compared to deep RRBS sequencing and conventional 
epigenetic clock analyses, this application of scAge reflects an up to 1000-fold reduction in sequence read numbers needed to assess epigenetic age. As such, this approach, in tandem with parallel library preparation workflows and optimized multiplexing strategies, may be applicable to large-scale data such as genetic screens, interventions studies and biobanks. Accordingly, these approaches may have important implications for clinical testing and in consumer settings. Overall, we present a novel computational approach which may enable accurate, rapid and cost-effective epigenetic age profiling from low-pass sequencing approaches.

\section{Methods}

\section{Animal resources and mouse models}

Mice originating in the Petkovich et al. study $^{10}$, with the exception of Snell dwarf, GHRKO models and 3-5-week-old C57BL/6 mice, were obtained from the NIA Aged Rodent Colony. The oldest mice obtained from NIA were 32 months; to obtain mice 34 and 35 months of age, 32-month-old mice were aged at Brigham \& Women's Hospital (BWH) for 2 and 3 months, respectively. With the exception of mice at the 34- and 35-month time points, blood from all mice used was collected within 2 weeks of arrival from NIA. Snell dwarf and GHRKO models and their controls were housed at the University of Michigan. The 3-5-week-old C57BL/6 mice were bred at BWH, Harvard Medical School, from parents obtained from NIA. Calorie restriction started at 14 weeks of age and continued until the time the animals were sacrificed. For all other animals in the Petkovich et al. study, food was provided ad libitum. All mouse data used from the Thompson et al. study ${ }^{27}$ were from ad libitum C57BL/6J mice housed at UCLA, which ranged in age from 2-21 months. 


\section{Downsampling methylation data}

Methylation data were obtained from the GEO database for both the Petkovich et al. ${ }^{10}$ and Thompson et al. ${ }^{27}$ datasets, with accession numbers GSE80672 ${ }^{10}$ and GSE120132 27 , respectively. Metadata was also downloaded from the GEO database using the GEOparse 2.0.3 package. Methylation sequences were mapped to the mm10/GRCm38 mouse genome, and methylation information was extracted by the original authors with Bismark ${ }^{39}$ in the case of the Petkovich et al. data, and BS-Seeker $2^{40}$ in the case of the Thompson et al. data. We further filtered processed methylation data to include only CpGs on autosomic chromosomes, in order to partially mitigate the effect of sex on predictions. Individual $\mathrm{CpG}$ reads (whether methylated or unmethylated) were concatenated into lists in a randomized order to prevent location bias from affecting downstream predictions. We then selected a defined number of reads ranging from 100 to 100,000 , each covering mostly unique CpGs, with some overlap when a larger number of reads were sampled. Methylation at $\mathrm{CpGs}$ in downsampled data was calculated as the mean of all methylation reads for that $\mathrm{CpG}$ in a particular subsample. Given the low number of reads, this most often produced binary methylation values ( 0 , unmethylated; 1 , methylated), with some additional fractional values in between this range that arose when multiple reads covered a single $\mathrm{CpG}$. In order to produce random subsamples, we used the sample function from the random Python library with five different reproducible seeds, enabling the generation of distinct assortments of CpGs for the same sample.

\section{Generating scAge reference models}

To create linear regression models enabling epigenetic age profiling via the scAge framework, we utilized deeply sequenced training data from the Petkovich et al. ${ }^{10}$ and Thompson et al. ${ }^{27}$ studies. 
Specifically, we filtered for only standard ad libitum blood C57BL/6J samples, resulting in dataframes with $\mathrm{n}=153$ and $\mathrm{n}=50$ samples for the Petkovich et al. ${ }^{10}$ and Thompson et al. ${ }^{27}$ studies, respectively. For each individual sample, only $\mathrm{CpG}$ sites covered 5 times or greater were retained, while other $\mathrm{CpG}$ sites were marked as missing. This filtering ensured high resolution estimation of methylation proportions in a particular sample, which is an integral component of the scAge training framework. Next, we progressively intersected all samples using an outer join methodology, capturing all CpGs covered 5x or more in at least one sample in the dataset. In order to select only CpGs which were consistently deeply covered across samples (facilitating accurate linear regression modeling), we removed $\mathrm{CpG}$ sites for which more than $10 \%$ of samples had missing values after depth filtration.

By applying these methods to both maximize $\mathrm{CpG}$ coverage and methylation value accuracy, we ultimately arrived at a table of 1,918,766 CpGs across 153 samples in the Petkovich et al. ${ }^{10}$ dataset and 1,202,751 CpGs across 50 samples in the Thompson et al. ${ }^{27}$ dataset. Of note, the Petkovich et al. ${ }^{10}$ dataset contained both negative and positive strand CpGs, while the Thompson et al. ${ }^{27}$ dataset contained only positive strand CpGs. This is a result of the processing methods used, wherein Thompson et al. ${ }^{27}$ concatenated positive and negative strand reads to the positive strand to increase confidence in the methylation values while decreasing the total feature space. This difference in processing, combined with significant batch effects, may help to explain the deviations that we observe when comparing predictions based on each reference model.

\section{Applying the scAge prediction framework}

To assess epigenetic age in low-coverage bulk data, we could not utilize conventional mouse methylation clocks, nor could we train novel methylation clocks based on classical elastic-net regression approaches. This is entirely due to the primary limitations of downsampled data: the 
lack of consistent $\mathrm{CpG}$ coverage across samples, coupled with a primarily binary data type. In order to overcome these fundamental constraints, we made use of the recently developed scAge framework, which enables accurate epigenetic age profiling in single cells ${ }^{26}$. Single-cell data features notoriously sparse, binary methylation profiles as a function of current limitations in sequencing protocols, which happen to heavily resemble downsampled bulk RRBS data. However, while the binary nature of single-cell methylation profiles is consistent with the biology underlying this data, bulk methylation profiles often contain fractional methylation values within the unit region, representing the proportion of cells in a specific sample that are methylated at a particular cytosine. Of note, these changes in fractional DNA methylation across bulk samples have been particularly key to methylation clock development in the past.

Practically, downsampled data were first intersected on an individual basis with a particular reference set. Next, of the common CpG sites between any subsample and the reference, a defined number (varied as a parameter in the analyses) of the most age-associated CpG sites-based on the magnitude of Pearson correlations - was chosen. This enabled testing distinct combinations of three critical parameters at once: the number of downsampled reads, the number of CpGs included in the scAge likelihood profile, and the particular random subsample. Among selected ageassociated $\mathrm{CpG}$ sites, the distance between the observed methylation value and the linear regression estimate for a particular cytosine was treated as a probability measure. In essence, we reasoned that the closer the observed methylation value was to the linear regression estimate for a particular age, the higher the probability of observing this methylation state at this age. Hence, we subtracted the distance between the observed value and the linear estimate from 1 , then proceeded to take the natural logarithm of the resulting difference to circumvent underflow errors in downstream processing. Once all relevant $\mathrm{CpG}$ sites were assayed for a particular age, we summed 
logarithmic probabilities together for the entire profile (equivalent to taking the product of all probabilities), generating a single value describing the likelihood of observing a particular limited methylome for each age. An age-likelihood distribution was hence created, mapping probabilities between -20 months and 60 months in high-resolution intervals of 0.1 months, ultimately enabling prediction of epigenetic age based on maximum likelihood estimation.

\section{Computational and statistical analyses}

All analyses were performed using Python 3.9.2, running with numpy 1.20.2 and pandas 1.2.4 for mathematical computing and data wrangling. Figures were generated using matplotlib 3.4.1 in combination with seaborn 0.11.1. Welch's one-tailed t-test assuming unequal variances, implemented in statannot 0.2 .3 and scipy 1.6.3, was used to perform statistical tests between groups. Pearson correlation coefficients and associated two-tailed p-values were computed using the pearsonr function implemented in the scipy package. Linear regressions underlying the scAge framework were computed with the LinearRegression function implemented in scikit-learn 0.24.2. Delta ages were computed as the difference between epigenetic age and chronological age. 


\section{Acknowledgments}

We are grateful to Csaba Kerepesi, Patrick Griffin, Yan $\mathrm{Hu}$, Marco Mariotti, Anastasia Shindyapina, Sun Hee Yim, Bohan Zhang, Benjamin Barre, Kejun “Albert” Ying, Jesse Poganik, Sang-Goo Lee, and Didac Santesmasses for helpful discussion. This work was supported by NIA grants to VNG. Some figures were created with BioRender.com.

\section{Author Contributions}

AT performed all analyses in the manuscript and developed the modified scAge framework. AT and VNG conceived the study and wrote the manuscript. VNG supervised the work.

\section{Data Availability}

All data used in this work were obtained from publicly available repositories. Bulk methylation data used for reference model training and downsampling was downloaded from GEO under accession numbers GSE120132 27 and GSE80672 ${ }^{10}$.

\section{Code Availability}

Source code for the modified scAge framework will be made available on GitHub.

\section{Competing Interests}

Brigham and Women's Hospital is the sole owner of a provisional patent application directed at this invention on which both AT and VNG are named inventors. 


\section{References}

1. Horvath, S. \& Raj, K. DNA methylation-based biomarkers and the epigenetic clock theory of ageing. Nat. Rev. Genet. 19, 371-384 (2018).

2. López-Otín, C., Blasco, M. A., Partridge, L., Serrano, M. \& Kroemer, G. The Hallmarks of Aging. Cell 153, 1194-1217 (2013).

3. Lister, R. et al. Human DNA methylomes at base resolution show widespread epigenomic differences. Nature 462, 315-322 (2009).

4. Meissner, A. et al. Reduced representation bisulfite sequencing for comparative highresolution DNA methylation analysis. Nucleic Acids Res. 33, 5868-5877 (2005).

5. Han, Y. et al. Targeted methods for epigenetic age predictions in mice. Sci. Rep. 10, 22439 (2020).

6. Han, Y. et al. New targeted approaches for epigenetic age predictions. BMC Biol. 18, 71 (2020).

7. Horvath, S. DNA methylation age of human tissues and cell types. Genome Biol. 14, 3156 (2013).

8. Smallwood, S. A. et al. Single-cell genome-wide bisulfite sequencing for assessing epigenetic heterogeneity. Nat. Methods 11, 817-820 (2014).

9. Guo, H. et al. Single-cell methylome landscapes of mouse embryonic stem cells and early embryos analyzed using reduced representation bisulfite sequencing. Genome Res. 23, 21262135 (2013).

10. Petkovich, D. A. et al. Using DNA Methylation Profiling to Evaluate Biological Age and Longevity Interventions. Cell Metab. 25, 954-960.e6 (2017).

11. Meer, M. V., Podolskiy, D. I., Tyshkovskiy, A. \& Gladyshev, V. N. A whole lifespan mouse multi-tissue DNA methylation clock. eLife 7, e40675 (2018). 
12. Stubbs, T. M. et al. Multi-tissue DNA methylation age predictor in mouse. Genome Biol. 18, 68 (2017).

13. Wang, T. et al. Epigenetic aging signatures in mice livers are slowed by dwarfism, calorie restriction and rapamycin treatment. Genome Biol. 18, 57 (2017).

14. Hannum, G. et al. Genome-wide methylation profiles reveal quantitative views of human aging rates. Mol. Cell 49, 359-367 (2013).

15. Galkin, F., Mamoshina, P., Kochetov, K., Sidorenko, D. \& Zhavoronkov, A. DeepMAge: A Methylation Aging Clock Developed with Deep Learning. Aging Dis. 12, 1252-1262 (2021).

16. Levine, M. E. et al. An epigenetic biomarker of aging for lifespan and healthspan. Aging 10, 573-591 (2018).

17. Fahy, G. M. et al. Reversal of epigenetic aging and immunosenescent trends in humans. Aging Cell 18, e13028 (2019).

18. Lu, Y. et al. Reprogramming to recover youthful epigenetic information and restore vision. Nature 588, 124-129 (2020).

19. Arneson, A. et al. A mammalian methylation array for profiling methylation levels at conserved sequences. bioRxiv 2021.01.07.425637 (2021) doi:10.1101/2021.01.07.425637.

20. Li, C. Z. et al. Epigenetic predictors of maximum lifespan and other life history traits in mammals. bioRxiv 2021.05.16.444078 (2021) doi:10.1101/2021.05.16.444078.

21. Haghani, A. et al. DNA Methylation Networks Underlying Mammalian Traits. bioRxiv 2021.03.16.435708 (2021) doi:10.1101/2021.03.16.435708.

22. Consortium, M. M. et al. Universal DNA methylation age across mammalian tissues. bioRxiv 2021.01.18.426733 (2021) doi:10.1101/2021.01.18.426733. 
23. Lu, A. T. et al. DNA methylation GrimAge strongly predicts lifespan and healthspan. Aging 11, 303-327 (2019).

24. Du, P. et al. Comparison of Beta-value and M-value methods for quantifying methylation levels by microarray analysis. BMC Bioinformatics 11, 587 (2010).

25. Infinium Mouse Methylation BeadChip | High-resolution epigenetic analyses of mouse strains. https://www.illumina.com/products/by-type/microarray-kits/infinium-mousemethylation.html.

26. Trapp, A., Kerepesi, C. \& Gladyshev, V. N. Profiling epigenetic age in single cells. bioRxiv 2021.03.13.435247 (2021) doi:10.1101/2021.03.13.435247.

27. Thompson, M. J. et al. A multi-tissue full lifespan epigenetic clock for mice. Aging 10, 2832-2854 (2018).

28. Wang, M. \& Lemos, B. Ribosomal DNA harbors an evolutionarily conserved clock of biological aging. Genome Res. 29, 325-333 (2019).

29. Zou, H. \& Hastie, T. Regularization and Variable Selection via the Elastic Net. J. R. Stat. Soc. Ser. B Stat. Methodol. 67, 301-320 (2005).

30. Angermueller, C., Lee, H. J., Reik, W. \& Stegle, O. DeepCpG: accurate prediction of singlecell DNA methylation states using deep learning. Genome Biol. 18, 67 (2017).

31. Kapourani, C.-A. \& Sanguinetti, G. Melissa: Bayesian clustering and imputation of singlecell methylomes. Genome Biol. 20, 61 (2019).

32. Kerepesi, C., Zhang, B., Lee, S.-G., Trapp, A. \& Gladyshev, V. N. Epigenetic clocks reveal a rejuvenation event during embryogenesis followed by aging. Sci. Adv. 7, eabg6082 (2021).

33. Kaeberlein, T. L. et al. Lifespan extension in Caenorhabditis elegans by complete removal of food. Aging Cell 5, 487-494 (2006). 
34. Fontana, L., Partridge, L. \& Longo, V. D. Extending healthy life span--from yeast to humans. Science 328, 321-326 (2010).

35. Takahashi, K. \& Yamanaka, S. Induction of pluripotent stem cells from mouse embryonic and adult fibroblast cultures by defined factors. Cell 126, 663-676 (2006).

36. Gill, D. et al. Multi-omic rejuvenation of human cells by maturation phase transient reprogramming. bioRxiv 2021.01.15.426786 (2021) doi:10.1101/2021.01.15.426786.

37. Flurkey, K., Papaconstantinou, J., Miller, R. A. \& Harrison, D. E. Lifespan extension and delayed immune and collagen aging in mutant mice with defects in growth hormone production. Proc. Natl. Acad. Sci. 98, 6736-6741 (2001).

38. Coschigano, K. T. et al. Deletion, but not antagonism, of the mouse growth hormone receptor results in severely decreased body weights, insulin, and insulin-like growth factor I levels and increased life span. Endocrinology 144, 3799-3810 (2003).

39. Krueger, F. \& Andrews, S. R. Bismark: a flexible aligner and methylation caller for Bisulfite-Seq applications. Bioinforma. Oxf. Engl. 27, 1571-1572 (2011).

40. Guo, W. et al. BS-Seeker2: a versatile aligning pipeline for bisulfite sequencing data. BMC Genomics 14, 774 (2013). 
Dio:Riv preprint doi: https://doi.org/10.1101/2021.10.25.465778; this version posted October 28, 2021. The copyright holder for this preprint FIg Ur hio was not certified by peer review) is the author/funder, who has granted bioRxiv a license to display the preprint in perpetuity. It is made available under aCC-BY-NC-ND 4.0 International license.

a

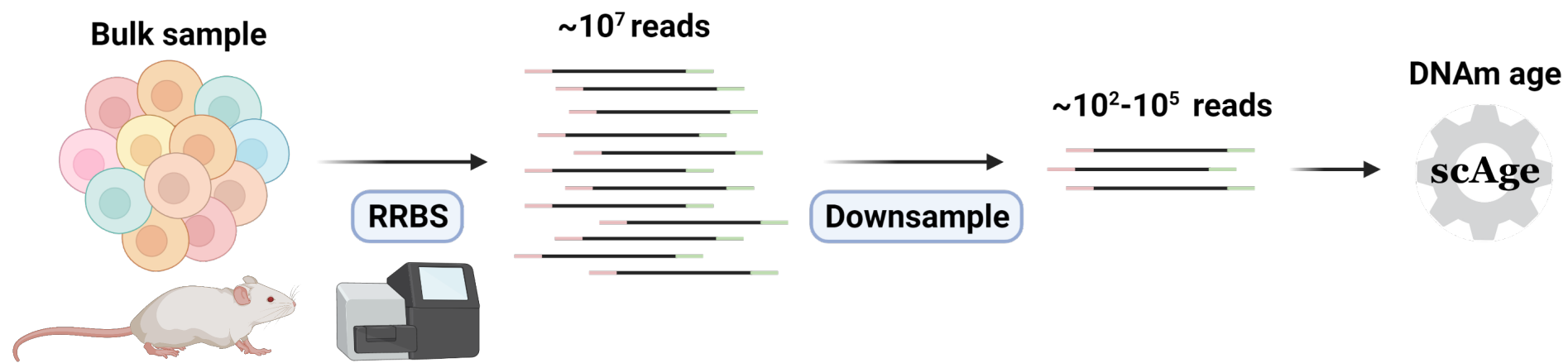

b
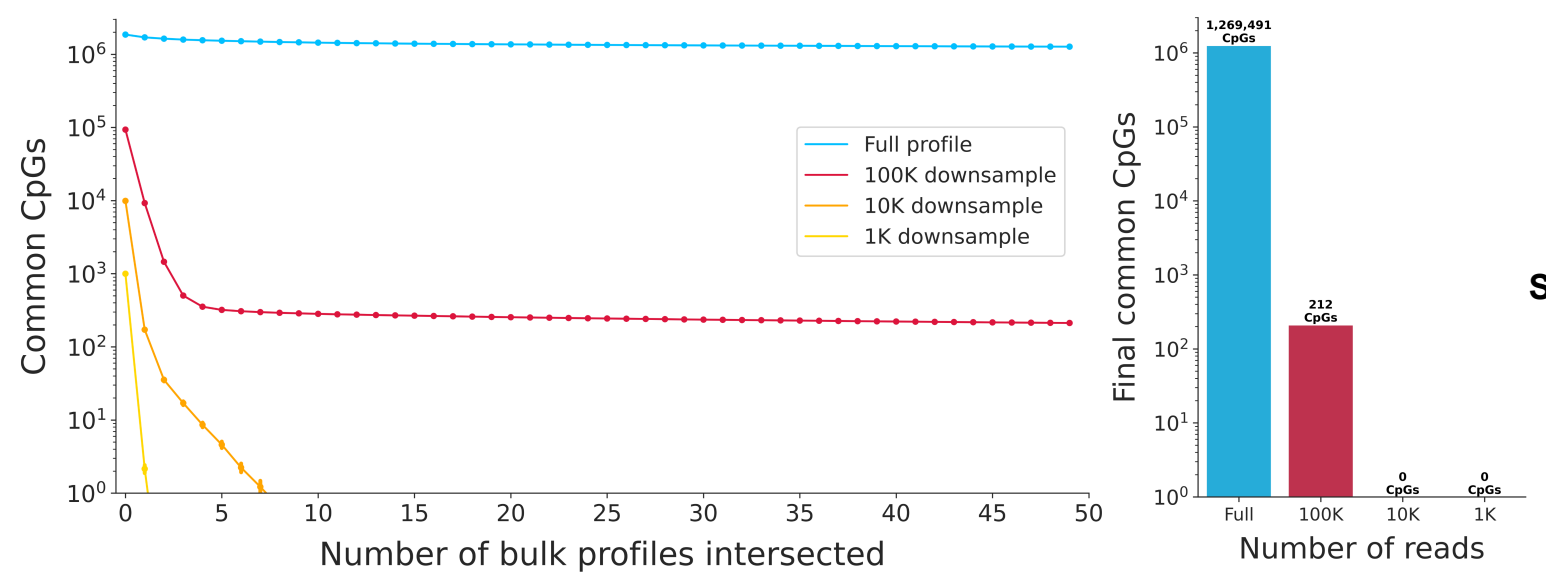

C

\begin{tabular}{ll|l|l|} 
Full: & $\mathrm{CPG} 1$ & $\mathrm{CPG} 2$ & $\mathrm{CPG} 3$ \\
\hline
\end{tabular}

\begin{tabular}{|c|c|c|c|}
\hline $\begin{array}{c}\text { Sample } \\
\text { A }\end{array}$ & 0.95 & 0.45 & 0.15 \\
\hline $\begin{array}{c}\text { Sample } \\
\text { B }\end{array}$ & 0.87 & 0.65 & 0.23 \\
\hline $\begin{array}{c}\text { Sample } \\
\text { C }\end{array}$ & 0.34 & 0.77 & 0.48 \\
\hline
\end{tabular}

\begin{tabular}{ll|l|l} 
Shallow: & $\mathrm{CPG} 1$ & $\mathrm{CPG} 2$ & $\mathrm{CPG} 3$ \\
\hline
\end{tabular}

\begin{tabular}{|c|c|c|c|}
$\begin{array}{c}\text { Sample } \\
\text { A }\end{array}$ & 0 & NA & 0.5 \\
\hline $\begin{array}{c}\text { Sample } \\
\text { B }\end{array}$ & NA & 1 & NA \\
\hline $\begin{array}{c}\text { Sample } \\
\text { C }\end{array}$ & 1 & NA & 0 \\
\hline
\end{tabular}

d

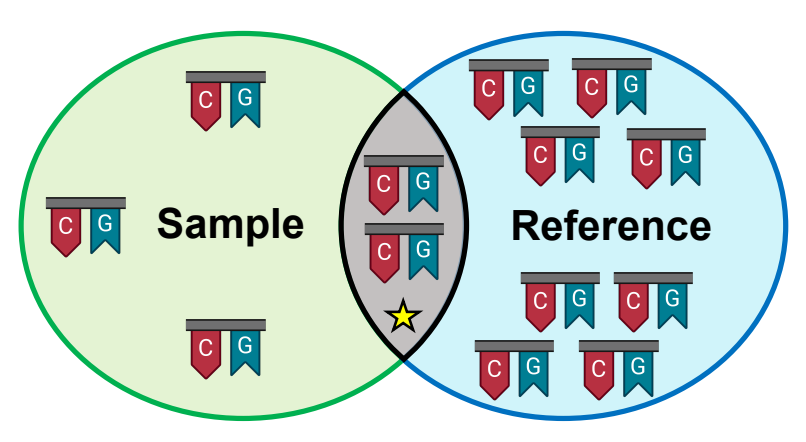

f

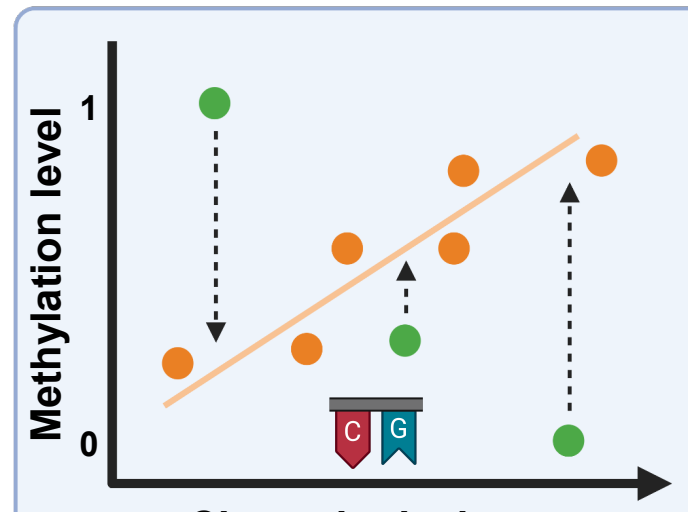

Chronological age
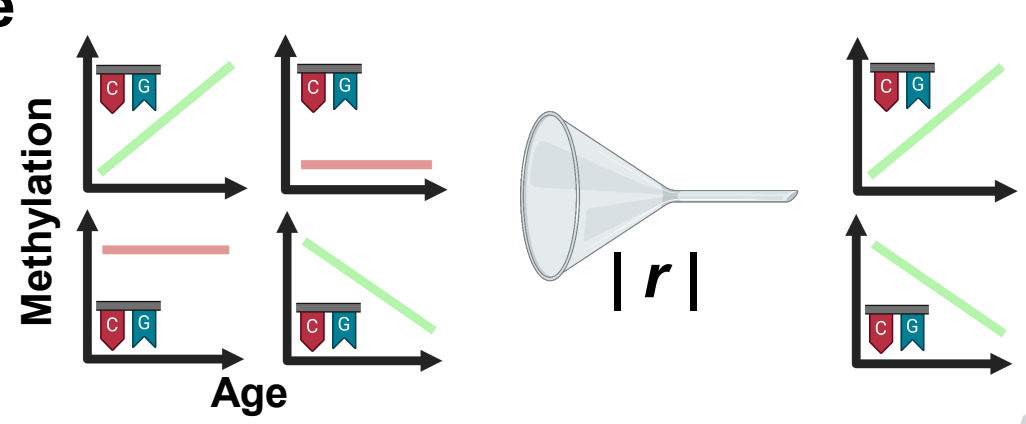

g
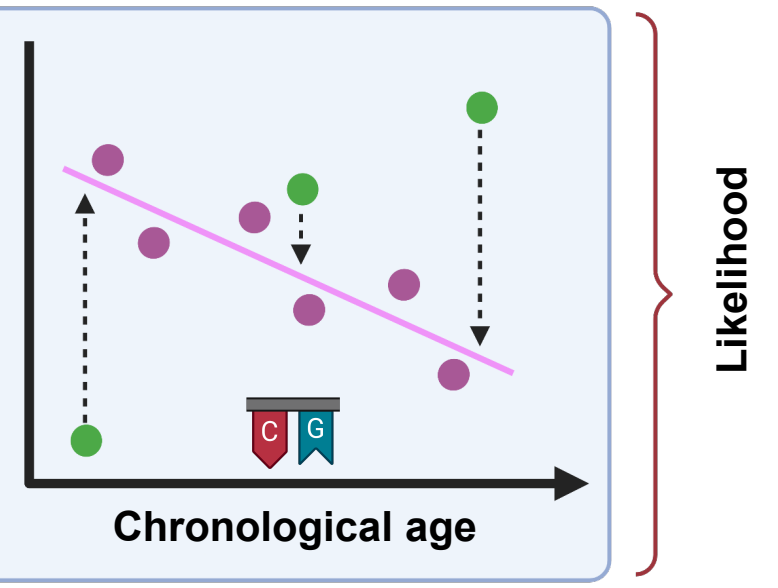

\section{Epigenetic age}




\section{Figure 1: An approach for low-pass epigenetic age profiling}

a) Schematic of the analysis pipeline. Murine bulk samples were collected and RRBS libraries were prepared and sequenced to a high depth ( $>10^{7}$ reads). Processed methylation data was downsampled with a reproducible random seed to $10^{2}-10^{5}$ reads, resulting in limited $\mathrm{CpG}$ profiles for each subsample. Lastly, epigenetic age ("DNAm age") was predicted by a modified version of the scAge framework.

b) Scatterplot (left) and barplot (right) representing the number of common $\mathrm{CpGs}$ after progressive intersections of full RRBS profiles as well as downsampled profiles $(100 \mathrm{~K}, 10 \mathrm{~K}$, and $1 \mathrm{~K}$ reads) in the Thompson et al. ${ }^{27}$ blood data. In the scatterplot, individual dots show the median of 100 permutations for each intersection, with each permutation producing a randomized intersection order. Y-axis is log-scale. Full RRBS profiles produce many intersections (blue), while downsampled profiles produce minimal to no intersections. Error bars depicting 95\% confidence intervals are shown, but in most cases are smaller than the size of the points. The barplot shows the final number of common $\mathrm{CpGs}$ for a particular profile size. c) Schematic tables highlighting the results of intersecting full RRBS profiles (blue, top) or downsampled profiles (red, bottom). With full profiles, many CpGs are covered consistently and at high depth across samples, resulting in valid fractional values within the unit region. However, with shallow (low-pass) sequencing, fewer reads are assessed in each sample, resulting in discordant $\mathrm{CpG}$ coverage, sparsity, and a primarily binary data type.

d) Intersection step of low-pass scAge, wherein only CpG sites common to both a sample and the reference dataset (highlighted by the star in the center) are retained for downstream processing.

e) Filtration step of low-pass scAge, wherein only common $\mathrm{CpG}$ sites exhibiting a robust positive/negative linear relationship between methylation level and age are chosen for downstream processing.

f) Probability computation step of low-pass scAge. Deeply sequenced samples are used as training data to construct linear regression models mapping changes in methylation as a function of chronological age. Individual orange/purple dots schematically depict training samples, and orange/purple lines depict ordinary least squares regressions based on these samples. Probability computations were performed by subtracting from 1 the distance from a particular observed $\mathrm{CpG}$ site methylation level in shallow data (green) to the linear regression estimate.

g) Likelihood distribution generated for a sample based on the collective probabilities harnessed across many CpGs. The dashed line depicts the age of maximum likelihood, which is interpreted as the epigenetic age of a particular sample. 
a
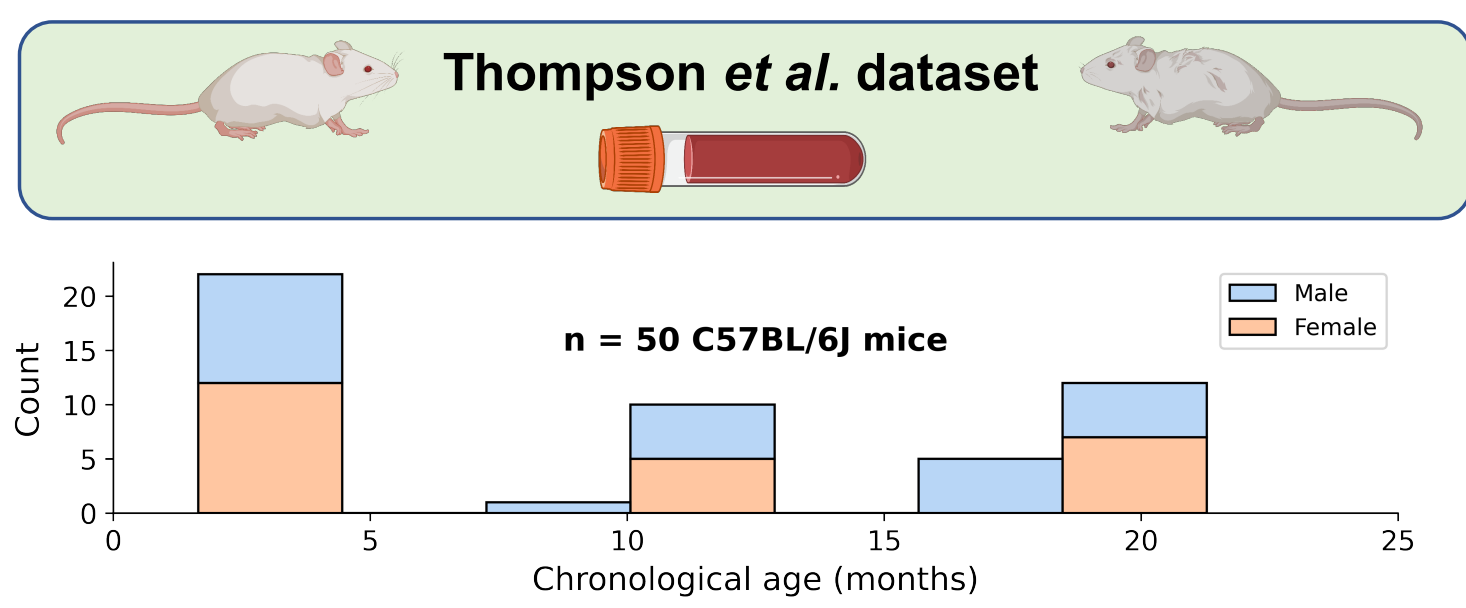

b

Thompson et al. models

Petkovich et al. models

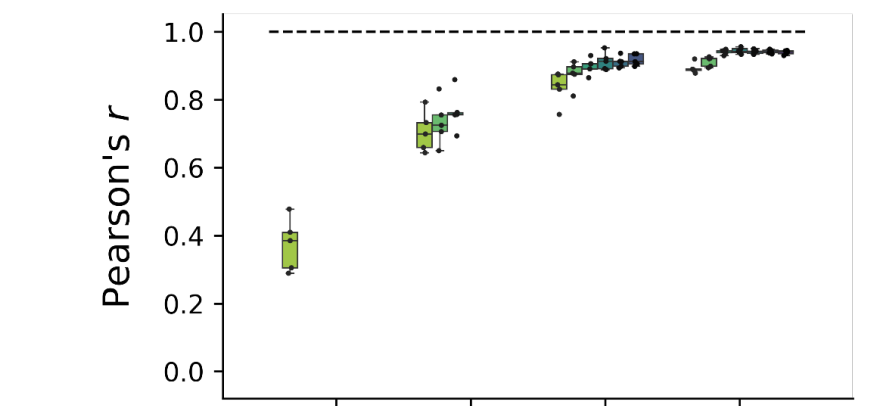

C

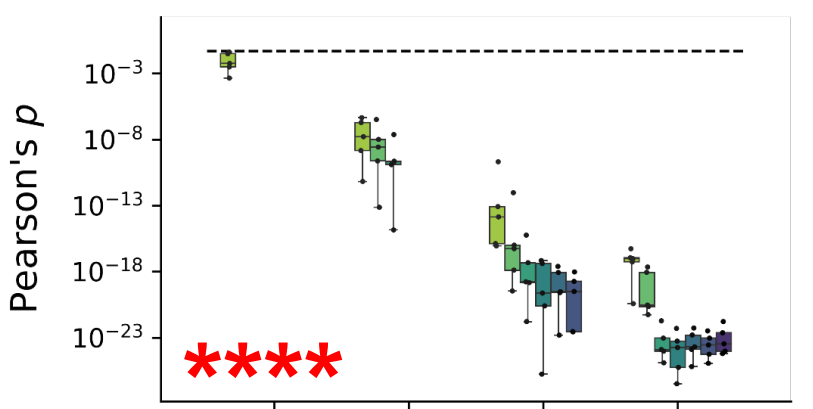

d

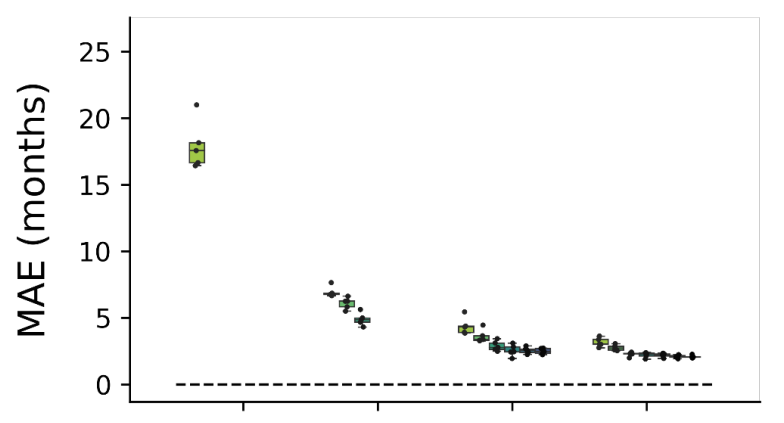

$* * * *$
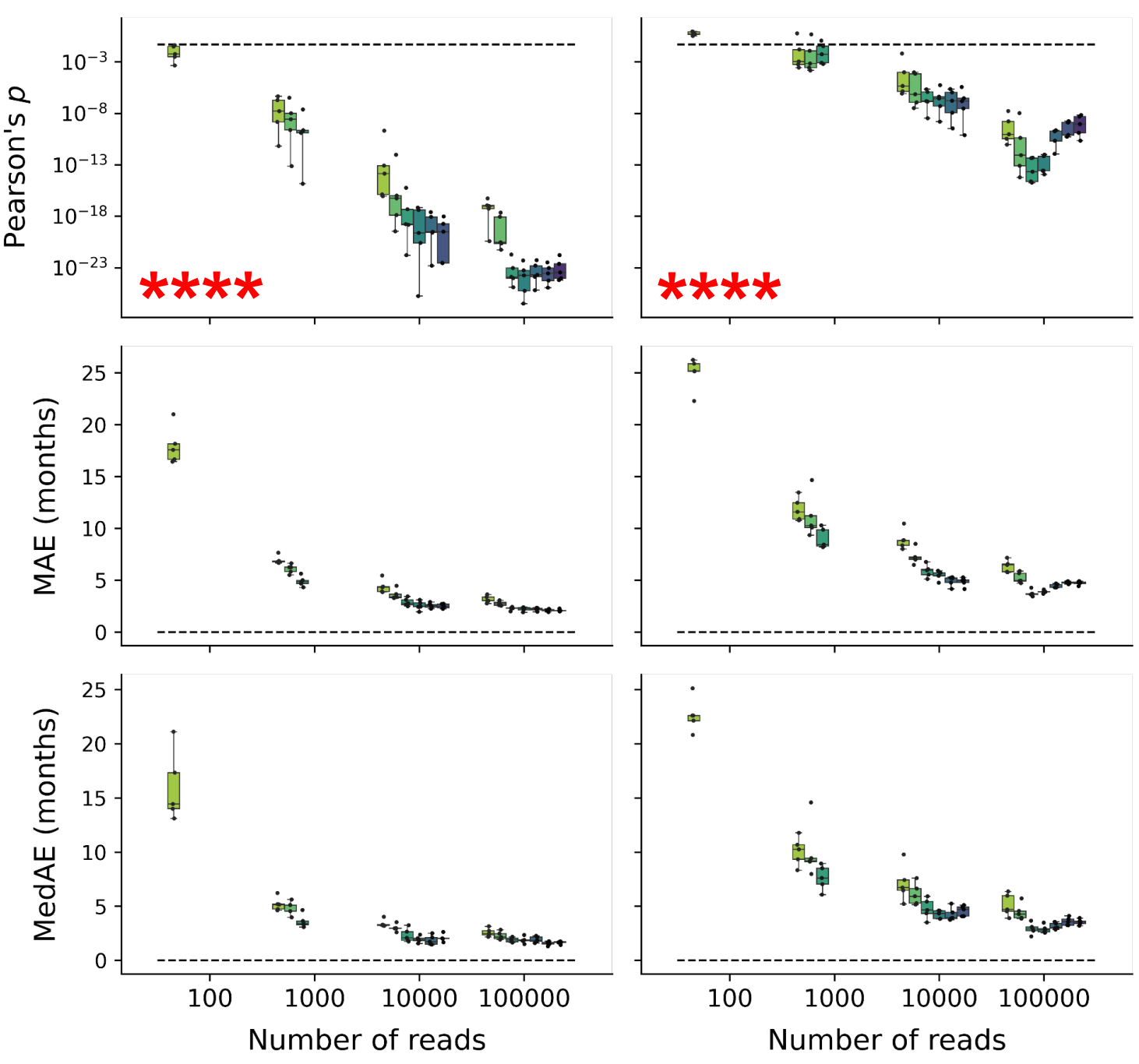

CpGs in scAge profile

$\square 50$

100

500

1000

2500

5000

10000

e

Number of reads 
Figure 2: Low-pass epigenetic age profiling of blood data from Thompson et al.

a) Schematic of the dataset (top) and age/sex distribution (bottom) of blood samples from C57BL/6J mice. Females $(n=24)$ are shown in orange, and males $(n=26)$ in blue. b-e) Boxplots of prediction metrics on the entirety of the Thompson et al. dataset. Predictions using Thompson et al. reference models are shown in the left panels, and predictions harnessing regression models built with the Petkovich et al. data are shown on the right. Individual dots (black) depict prediction metrics for a particular set of: 1) a random state 2) a number of downsampled reads, and 3) an scAge profiling parameter (the number of CpGs included in the age computation likelihood profile). Individual boxplots depict the median and the $1^{\text {st }}$ and $3^{\text {rd }}$ quartiles, with whiskers extending to $1.5 \mathrm{x}$ the interquartile range. Boxplots are colored based on the number of $\mathrm{CpGs}$ to profile in the likelihood computation. (b) depicts Pearson correlation coefficients, (c) depicts the associated two-tailed p-values, (d) depicts the mean absolute errors in months, and (e) depicts the median absolute errors in months. Red asterisks $(* * * *)$ highlight that significant $\mathrm{p}$-values below 0.0001 were reached. Dashed lines highlight $r=1, \mathrm{p}=0.05$ (nominal significance), $\mathrm{MAE}=0$, and $\mathrm{MedAE}=0$. 
bioßziv preprint doi: https://doi.org/10.1101/2021.10.25.465778; this version posted October 28,2021 . The copyright holder for this preprint FIO Ur his was not certified by peer review) is the author/funder, who has granted bioRxiv a license to display the preprint in perpetuity. It is made available under aCC-BY-NC-ND 4.0 International license.

a
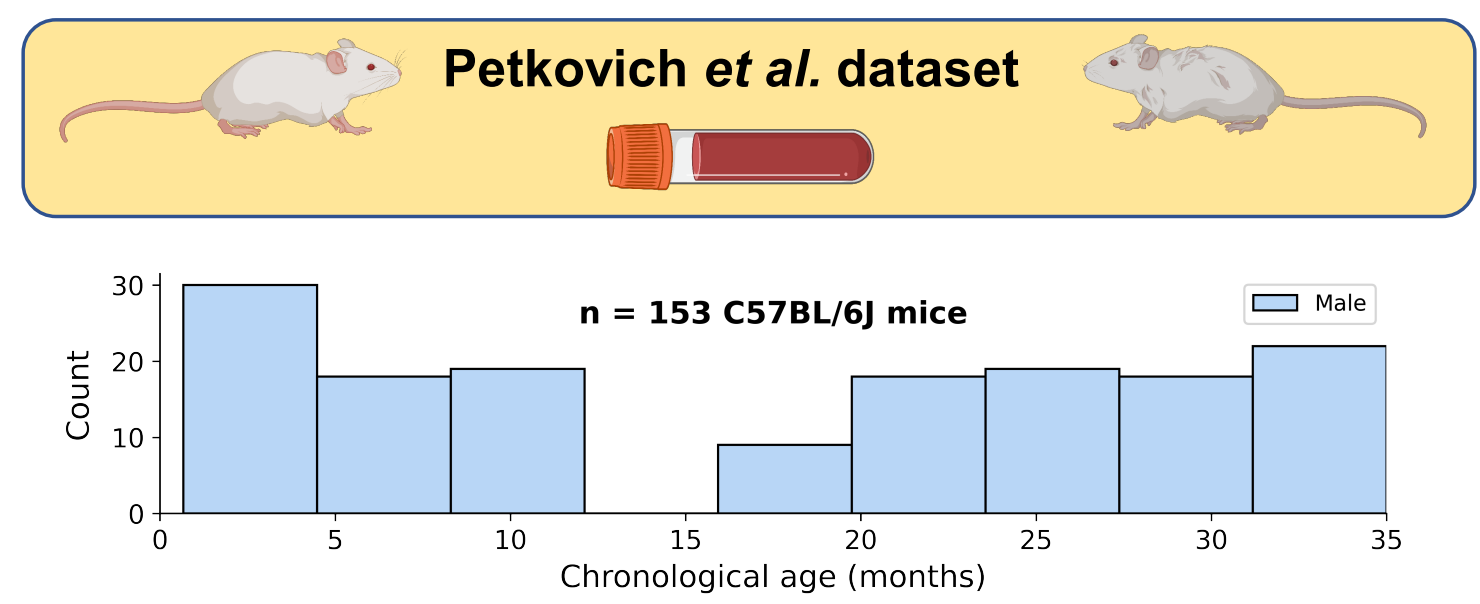

b

Thompson et al. models

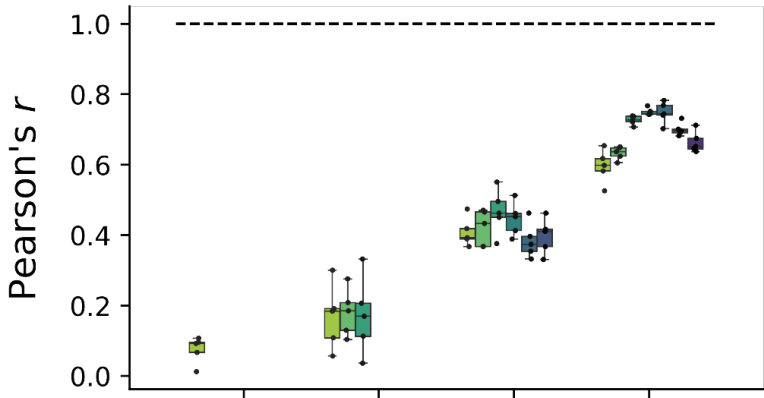

C

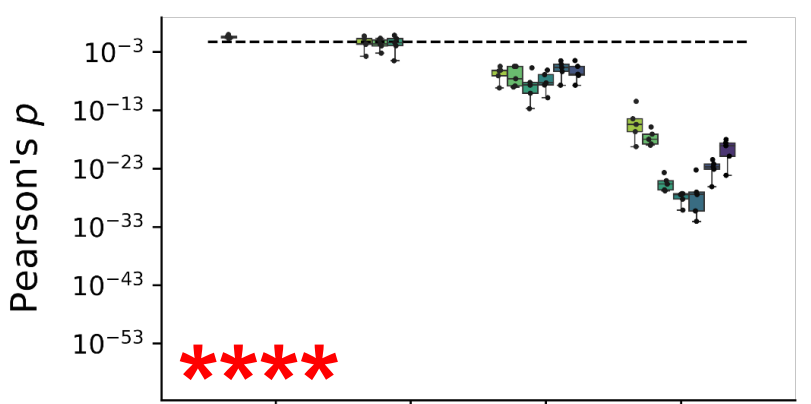

d

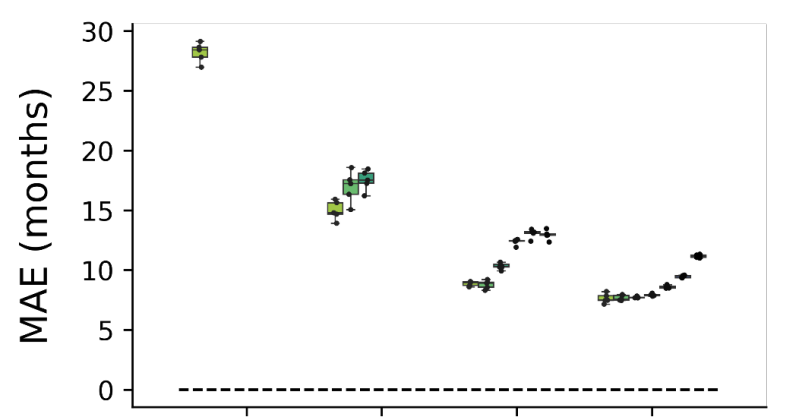

e

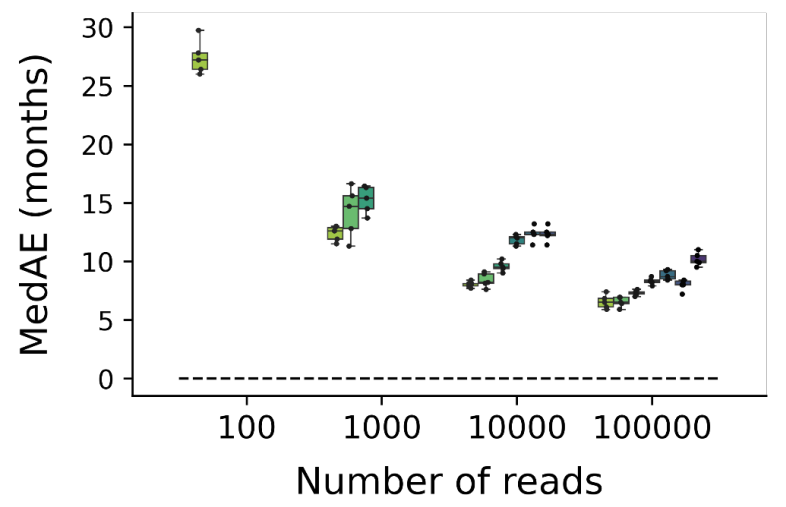

Petkovich et al. models
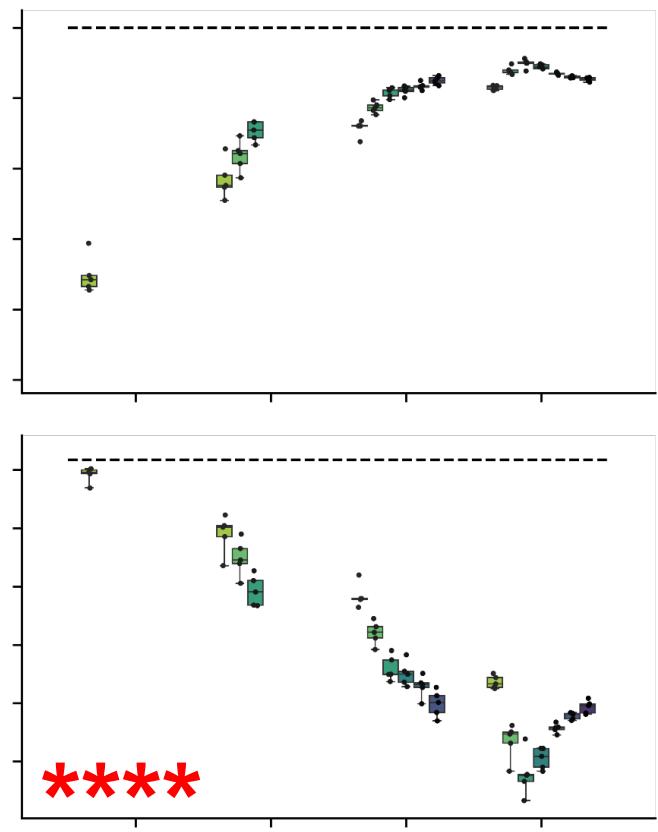

.

CpGs in scAge profile

$\square 0$

100

500

1000

2500

5000

10000

F

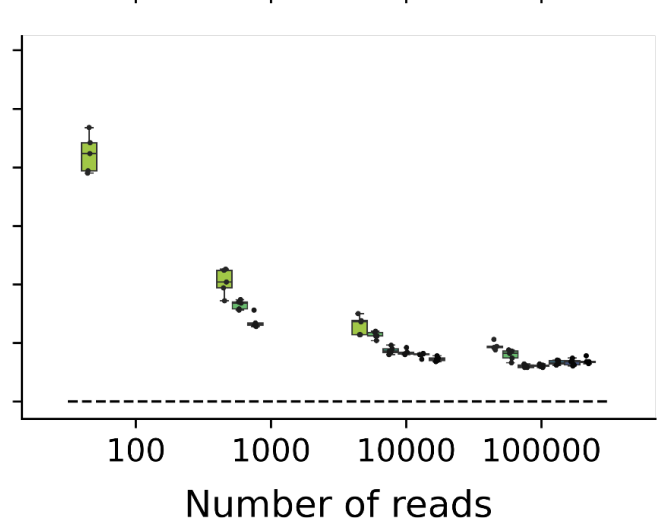




\section{Figure 3: Low-pass epigenetic age profiling of blood data from Petkovich et al.}

a) Schematic of the dataset (top) and age distribution (bottom) of blood samples from male C57BL/6J mice $(\mathrm{n}=153)$.

b-e) Boxplots of predictive metrics on the entirety of the Petkovich et al. dataset. Predictions using Thompson et al. reference models are shown in the left panels, and predictions harnessing regression models built with the Petkovich et al. data are shown on the right. Individual dots (black) depict prediction metrics for a particular set of: 1) a random state 2) a number of downsampled reads, and 3) a scAge profiling parameter (the number of CpGs included in the likelihood profile). Individual boxplots depict the median and the $1^{\text {st }}$ and $3^{\text {rd }}$ quartiles, with whiskers extending to $1.5 \mathrm{x}$ the interquartile range. Boxplots are colored based on the number of CpGs to profile in the likelihood computation. (b) depicts Pearson correlation coefficients, (c) depicts the associated two-tailed p-values, (d) depicts the mean absolute errors in months, and (e) depicts the median absolute errors in months. Red asterisks $(* * * *)$ highlight that significant $\mathrm{p}$ values below 0.0001 were reached. Dashed lines highlight $r=1, \mathrm{p}=0.05$ (nominal significance), $\mathrm{MAE}=0$, and $\mathrm{MedAE}=0$. 
DioPaiv preprint doi: https://doi.org/10.1101/2021.10.25.465778; this version posted October 28, 2021. The copyright holder for this preprint FIg Urahis was not certified by peer review) is the author/funder, who has granted bioRxiv a license to display the preprint in perpetuity. It is made available under aCC-BY-NC-ND 4.0 International license.
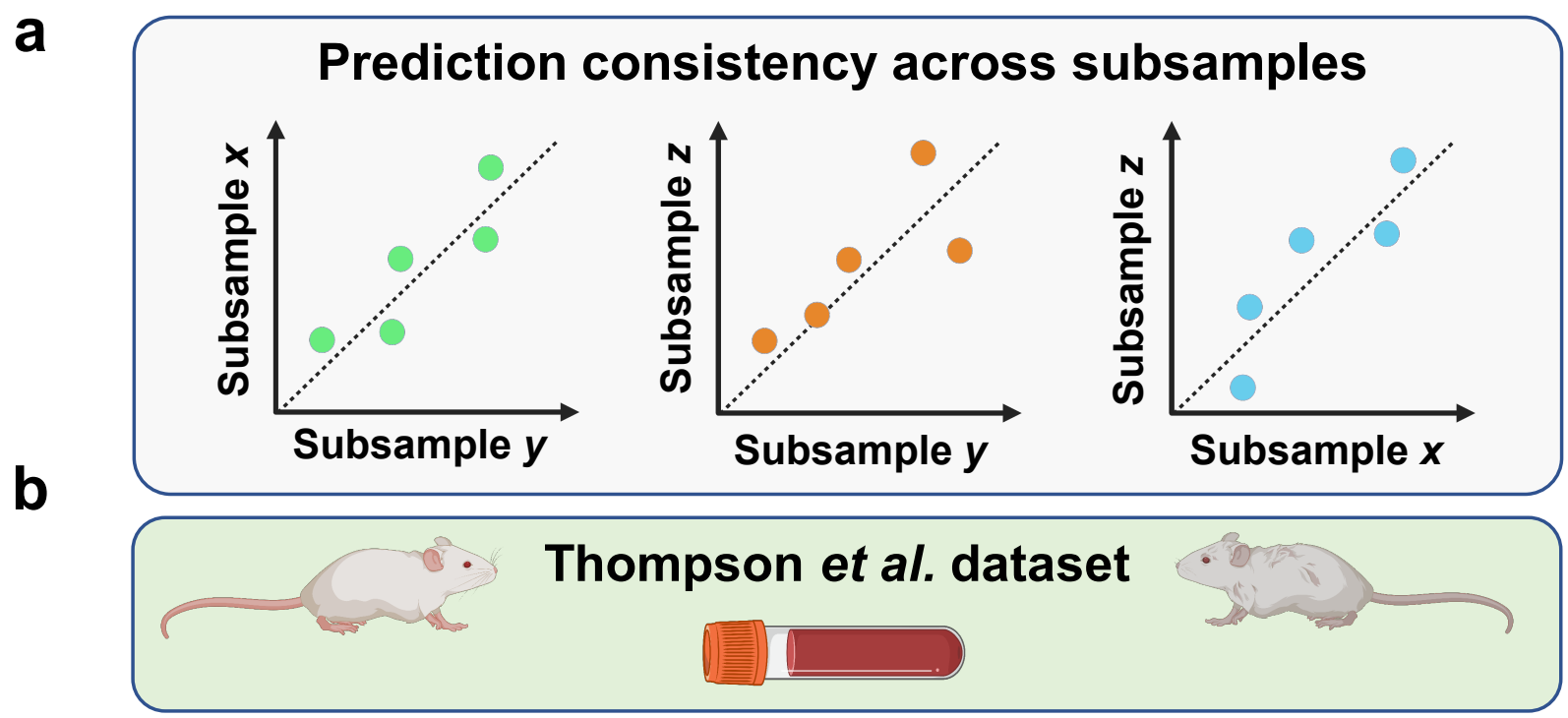

Thompson et al. models
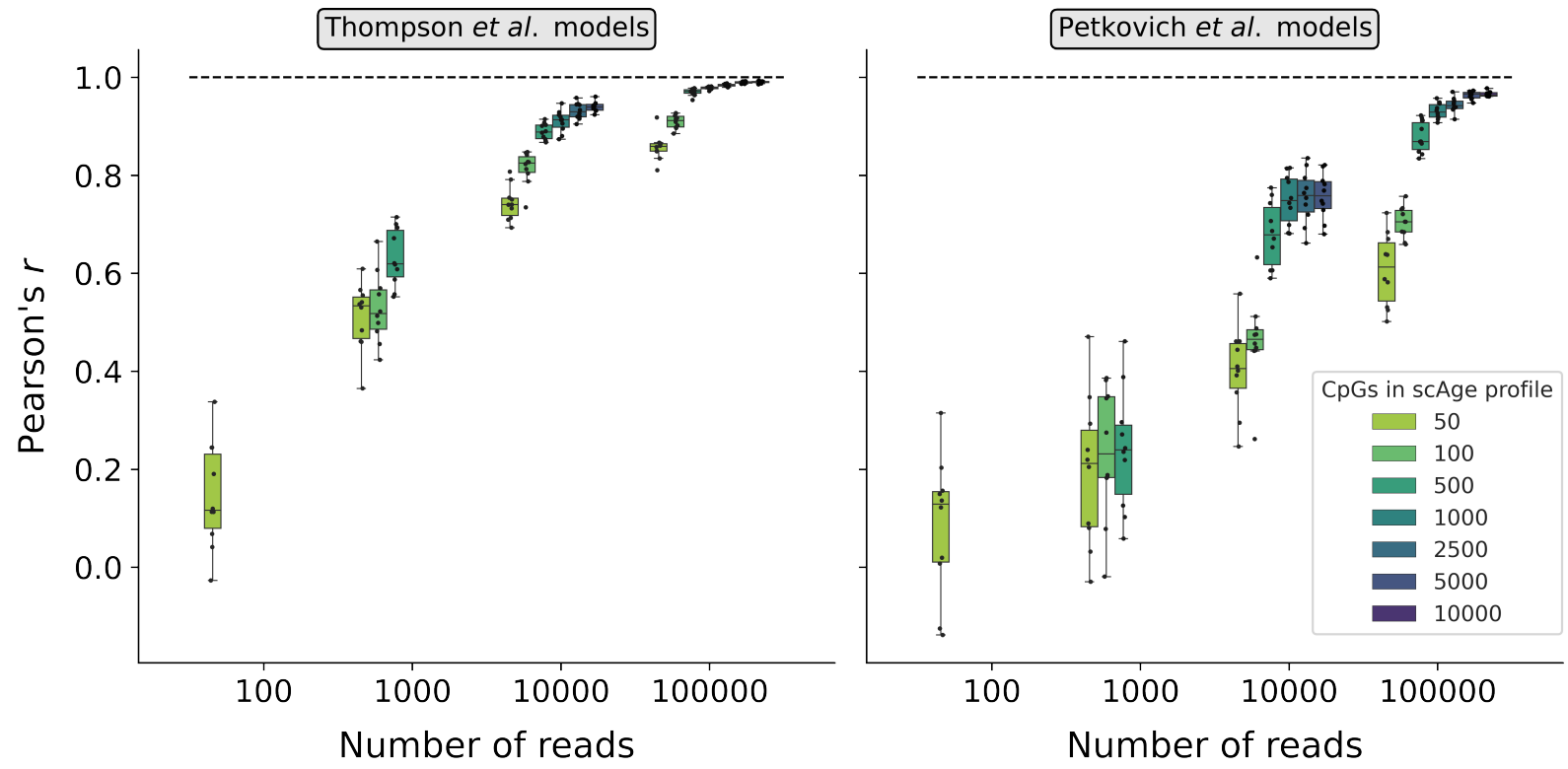

C
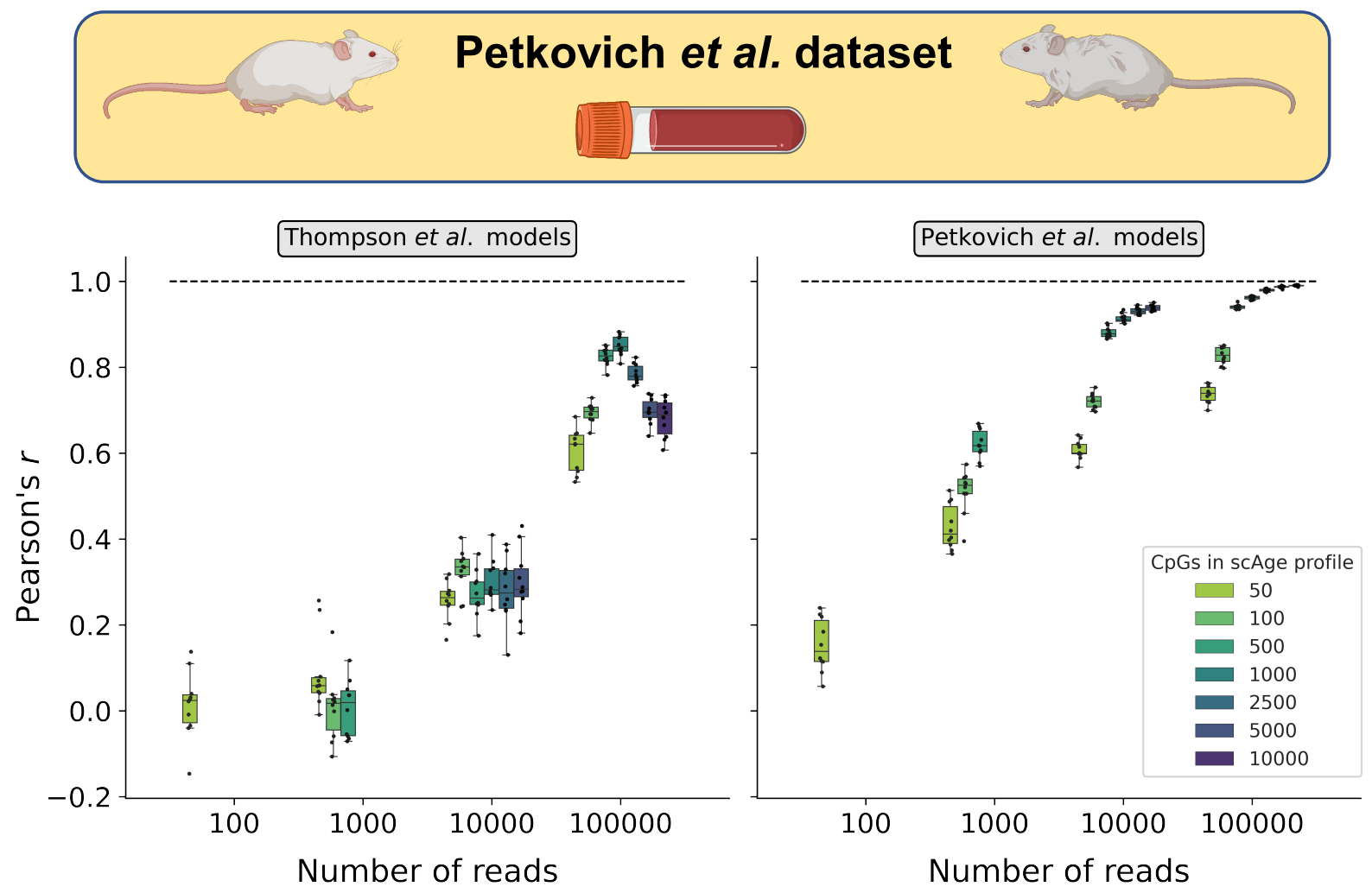
Figure 4: Consistency of low-pass epigenetic age predictions across random subsamples a) Schematic of the analyses presented in this figure. Epigenetic age predictions from different random subsamples are compared, and the linearity of their relationship is evaluated by Pearson correlation analysis.

b-c) Boxplots of inter-subsample Pearson correlation coefficients $(r)$ on the entirety of (b) the Thompson et al. dataset and (c) the Petkovich et al. dataset. Inter-subsample associations using the Thompson et al. reference models are shown in the left panels, and inter-subsample associations using the Petkovich et al. models are shown on the right. Individual dots (black) depict Pearson correlations between predicted epigenetic age across two different random seeds for the same sample; since 5 random seeds were utilized in total, 10 possible combinations of random seeds are possible $(\mathrm{n}=10$ dots/boxplot). Individual boxplots depict the median and the $1^{\text {st }}$ and $3^{\text {rd }}$ quartiles, with whiskers extending to $1.5 x$ the interquartile range. Boxplots are colored based on the scAge parameter used for these predictions (i.e., the number of $\mathrm{CpGs}$ to profile in the likelihood computation). The dashed lines in the boxplots highlight $r=1$. 
a

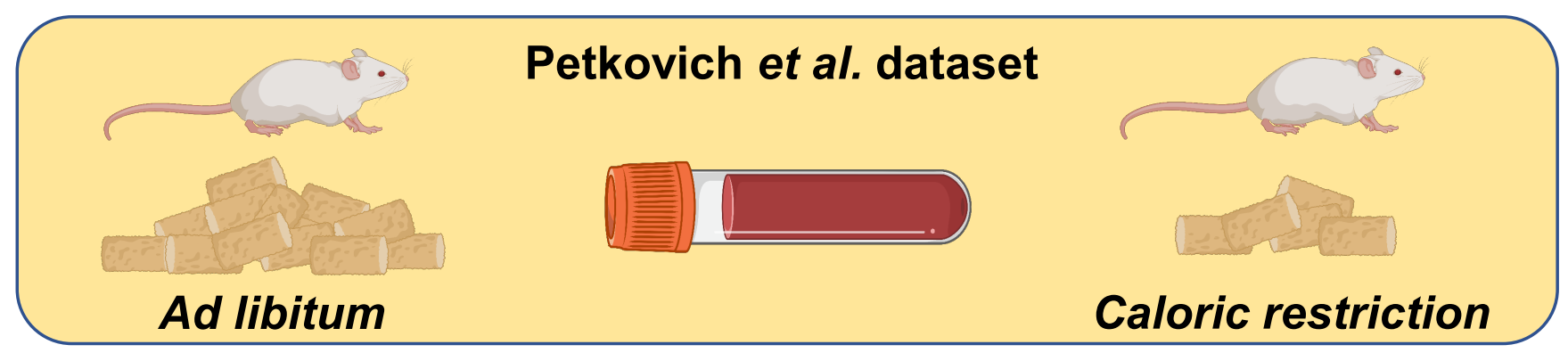

b
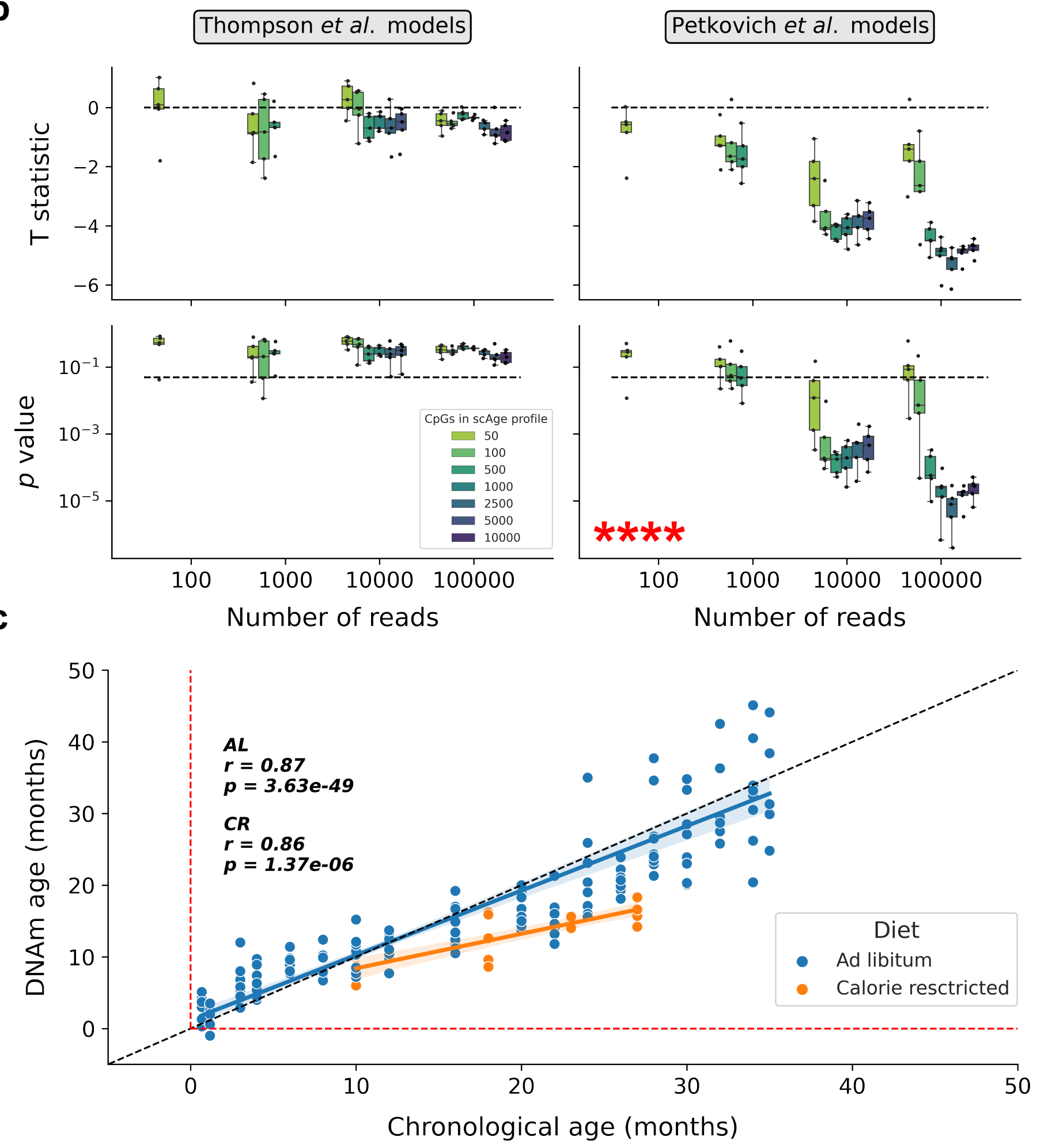
Figure 5: Attenuated epigenetic aging trajectories in response to calorie restriction a) Schematic outline of the analyses presented in this figure. Blood age predictions and trajectories in standard ad libitum $(\mathrm{n}=153)$ and calorie restricted $(\mathrm{n}=20)$ male C57BL/6J mice originating from the Petkovich et al. ${ }^{10}$ study were compared.

b) Boxplots of statistical testing metrics based on delta age measurements (epigenetic age chronological age). Significance testing metrics using the Thompson et al. reference models are shown in the left panels, and those using the Petkovich et al. models on the right. Individual dots (black) depict prediction metrics for a particular random state, number of downsampled reads, and scAge profiling parameter. Boxplots show the median and the $1^{\text {st }}$ and $3^{\text {rd }}$ quartiles, with whiskers extending to $1.5 \mathrm{x}$ the interquartile range. Boxplots are colored based on the scAge parameter used for these predictions (i.e., the number of $\mathrm{CpGs}$ to profile in the likelihood computation). Upper panels depict the T statistic from Welch's t-test used to quantify statistical significance between delta age in ad libitum and calorically restricted (CR) samples, with negative values indicating lower delta age in CR samples. Lower panels depict the one-tailed pvalues associated with these t-tests. Red asterisks (****) highlight that significant p-values below 0.0001 were reached. The dashed lines in the upper panels highlight $\mathrm{T}=0$, and the dashed lines in the lower panels highlight nominal significance $(p=0.05)$.

c) Scatterplot showing the relationship between epigenetic age and chronological age for ad libitum mice (blue, $\mathrm{n}=153$ ) and calorie-restricted mice (orange, $\mathrm{n}=20$ ) for one random state with 100,000 downsampled reads and the top 2,500 age-associated CpGs included in the likelihood profile. The dotted black line represents the identity line between chronological and DNAm age. Red dotted lines highlight DNAm and chronological ages of 0 months. The Pearson correlation coefficient $(r)$ and its associated two-tailed p-value is shown separately for each group in the upper left corner. Regression models (blue/orange) are shown with $95 \%$ confidence intervals (light blue/orange). 
bioRxiv preprint doi: https://doi.org/10.1101/2021.10.25.465778; this version posted October 28 , 2021. The copyright holder for this preprint FIg Urahi was not certified by peer review) is the author/funder, who has granted bioRxiv a license to display the preprint in perpetuity. It is made available under aCC-BY-NC-ND 4.0 International license.

a
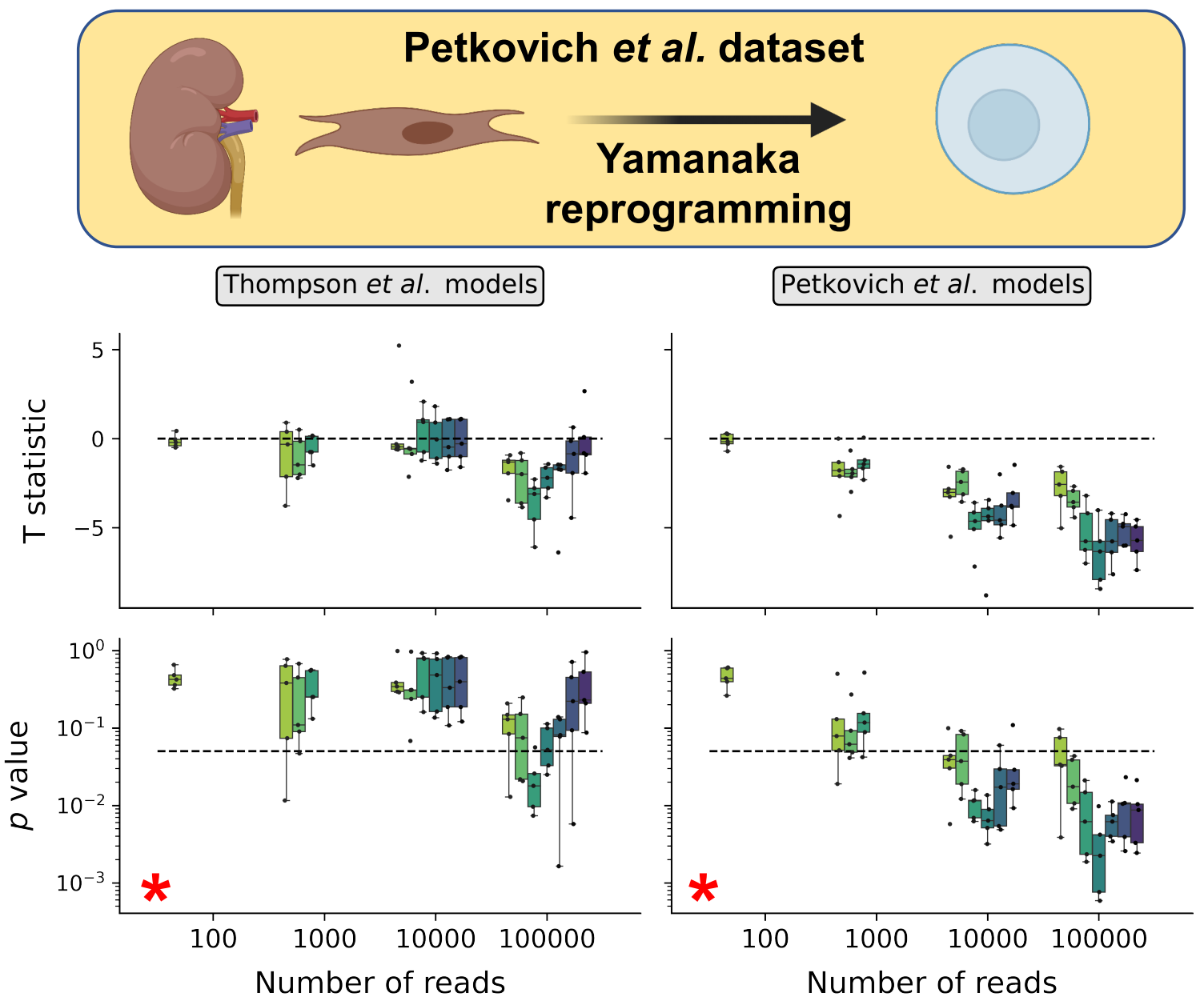

b
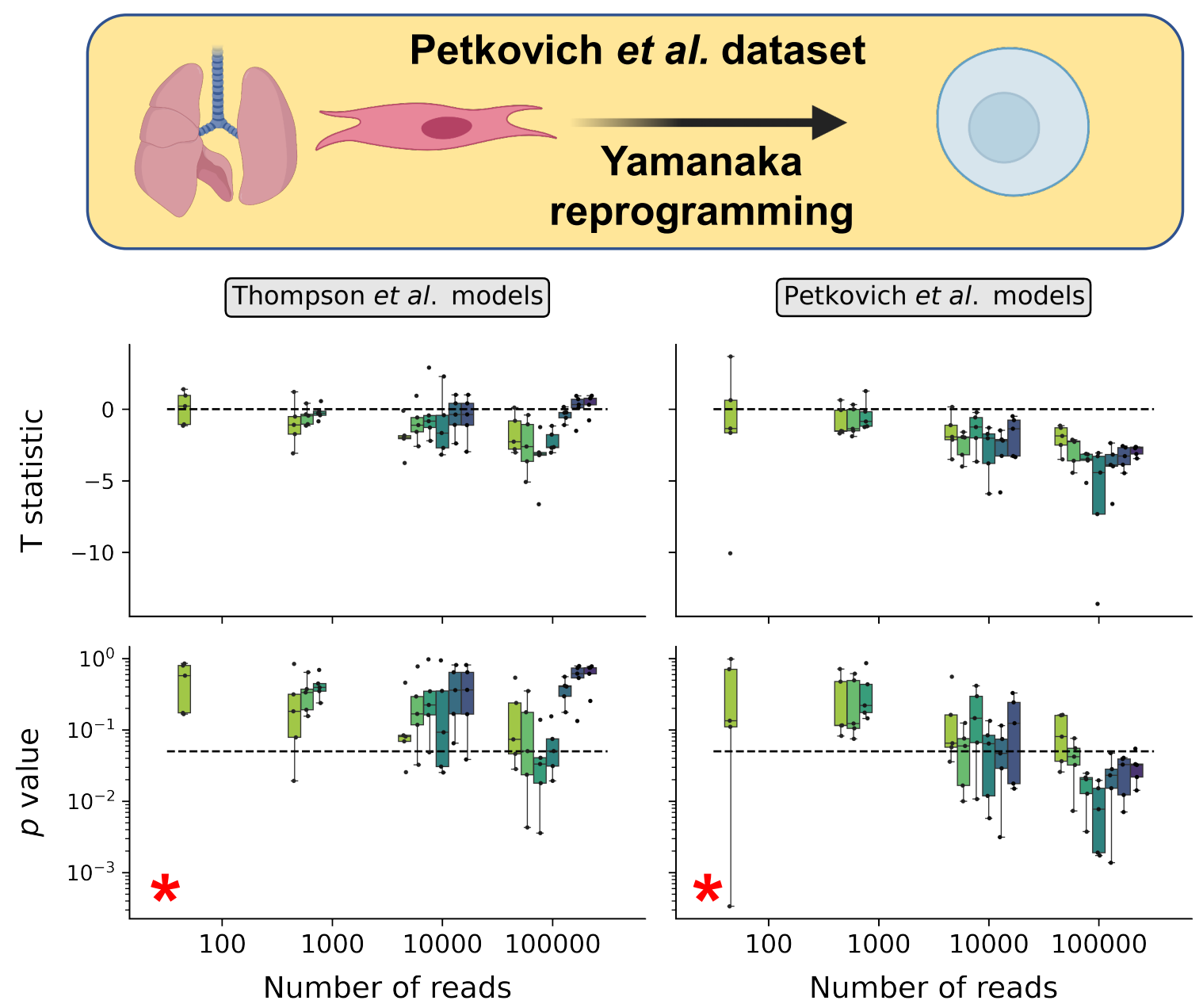

CpGs in scAge profile

$\square 50$

100

500

1000

2500

5000

10000

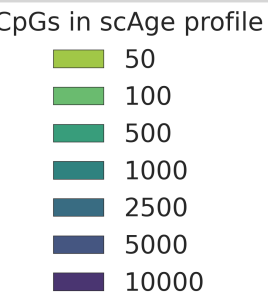




\section{Figure 6: Low-pass scAge tracks epigenetic age reversal by iPSC reprogramming}

a-b) Experimental schematics (top), and boxplots (bottom) of statistical testing metrics based on epigenetic age measurements in (a) renal fibroblasts and iPSC samples derived from these cells and (b) lung fibroblasts and iPSC samples derived from these cells, all from the Petkovich et al. ${ }^{10}$ study ( $\mathrm{n}=3$ per group). Significance testing metrics using the Thompson et al. reference models are shown in the left panels, and those using Petkovich et al. reference models are shown on the right. Individual dots (black) depict prediction metrics for a particular random state, number of downsampled reads, and scAge profiling parameter. Boxplots depict the median and the $1^{\text {st }}$ and $3^{\text {rd }}$ quartiles, with whiskers extending to $1.5 \mathrm{x}$ the interquartile range. Boxplots are colored based on the scAge parameter used for these predictions (i.e., the number of CpGs to profile in the likelihood computation). Upper plots in each panel depict the T statistic from Welch's t-test used to quantify statistical significance between epigenetic age in fibroblasts and iPSC samples, with negative values indicating lower average epigenetic age in iPSC samples. Lower panels depict the one-tailed p-values associated with these t-tests. Red asterisk $\left(^{*}\right)$ highlights that significant pvalues below 0.05 were reached. The dashed lines in the upper panels highlight $\mathrm{T}=0$, and the dashed lines in the lower panels depict nominal significance $(p=0.05)$. 
a
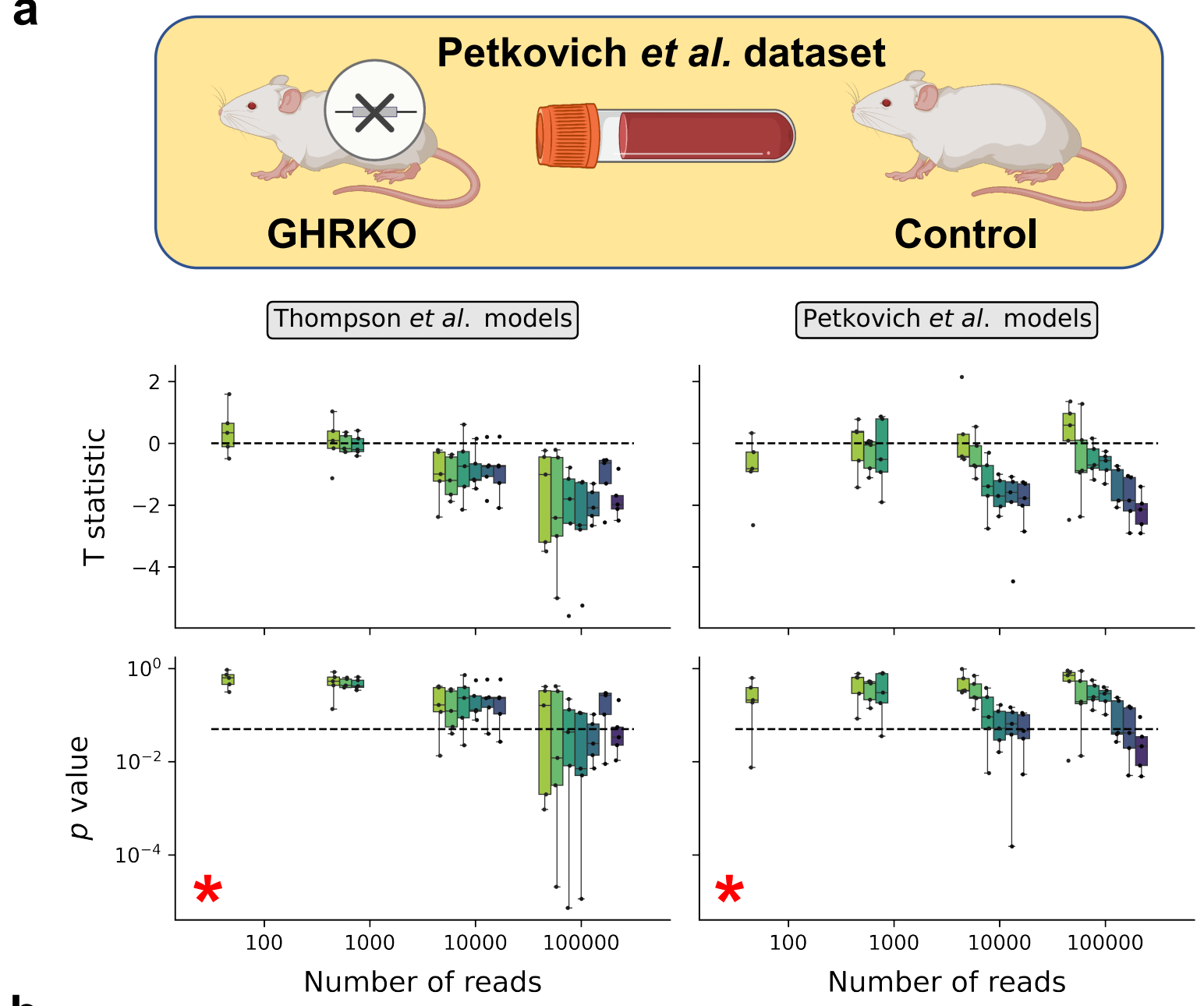

b

CpGs in scAge profile

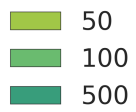

500

1000

2500

5000

10000
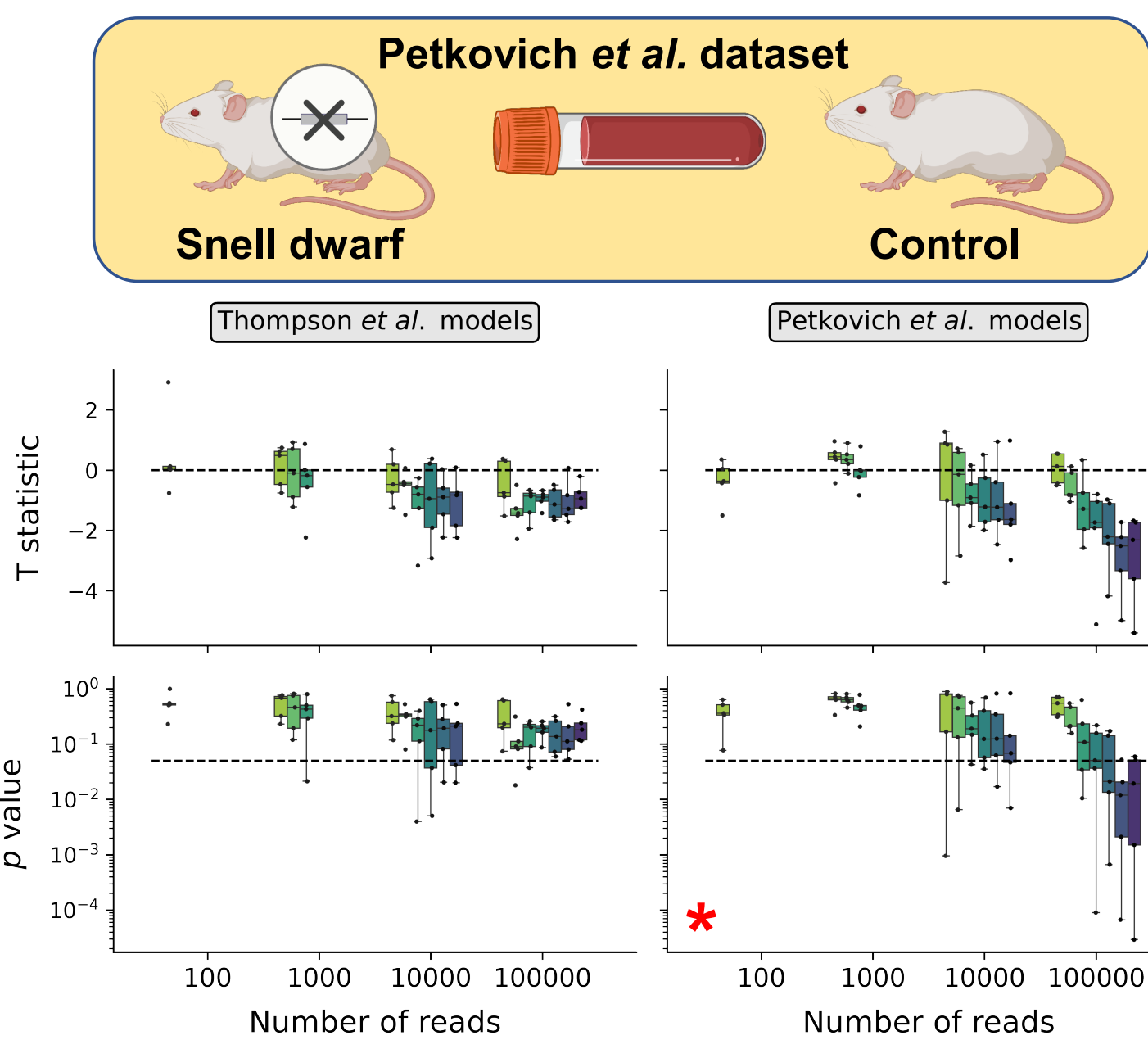

Petkovich et al. models

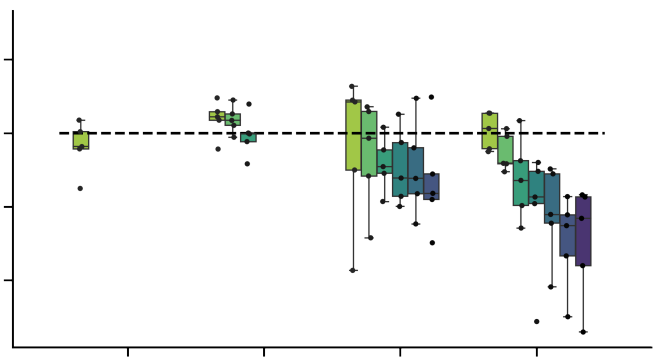

CpGs in scAge profile

$\square 50$

$\square 100$

500

- 1000

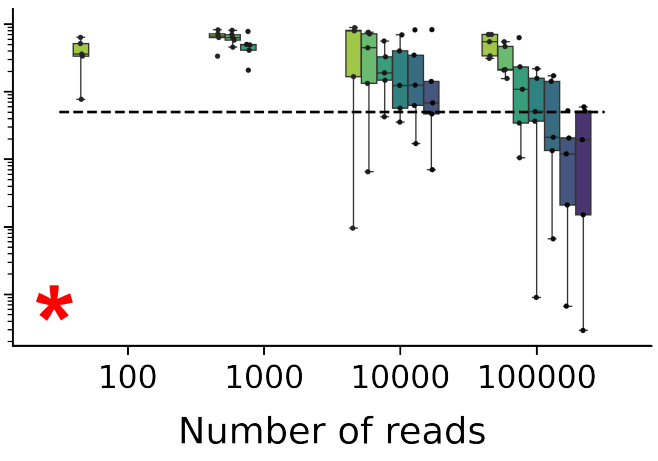

2500

5000

- 10000 


\section{Figure 7: Low-coverage profiling tracks reduction in age from genetic manipulations}

a-b) Experimental schematics (top), and boxplots (bottom) of statistical testing metrics based on epigenetic age measurements in $(\mathrm{a}) \mathrm{GRHKO}(\mathrm{n}=15)$ and wildtype C57BL/6J x BALB/cByJ)/F2 $(\mathrm{n}=11)$ blood samples of both sexes, aged 6 months and $(\mathrm{b})$ Snell dwarf $(\mathrm{n}=8)$ and wildtype $(\mathrm{DW} / \mathrm{J} \times \mathrm{C} 3 \mathrm{H} / \mathrm{HEJ}) / \mathrm{F} 2(\mathrm{n}=10)$ blood samples of both sexes, aged 6 months, from the Petkovich et al. ${ }^{10}$ study. Significance testing metrics using the Thompson et al. reference models are shown in the left panels, and those using the Petkovich et al. models are shown on the right. Individual dots (black) depict prediction metrics for a particular random state, number of downsampled reads, and scAge profiling parameter. Individual boxplots depict the median and the $1^{\text {st }}$ and $3^{\text {rd }}$ quartiles, with whiskers extending to $1.5 \mathrm{x}$ the interquartile range. Boxplots are colored based on the scAge parameter used for these predictions (i.e., the number of $\mathrm{CpGs}$ to profile in the likelihood computation). Upper plots in each panel depict the T statistic from Welch's t-test used to quantify statistical significance between epigenetic age in genetically altered and wild-type samples, with negative values indicating lower epigenetic age in GHRKO/Snell dwarf samples. Lower panels depict the one-tailed p-values associated with these t-tests. Red asterisk (*) highlights that significant $\mathrm{p}$-values below 0.05 were reached. The dashed lines in the upper panels mark $\mathrm{T}=0$, and the dashed lines in the lower panels highlight nominal significance $(\mathrm{p}=0.05)$. 


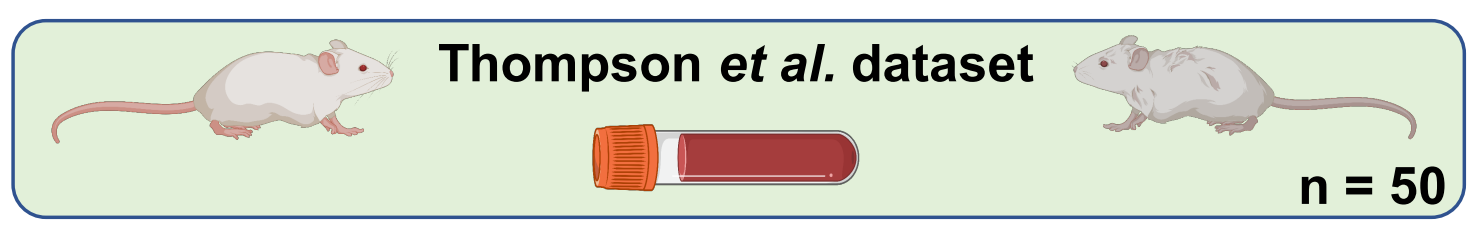

Thompson et al. models
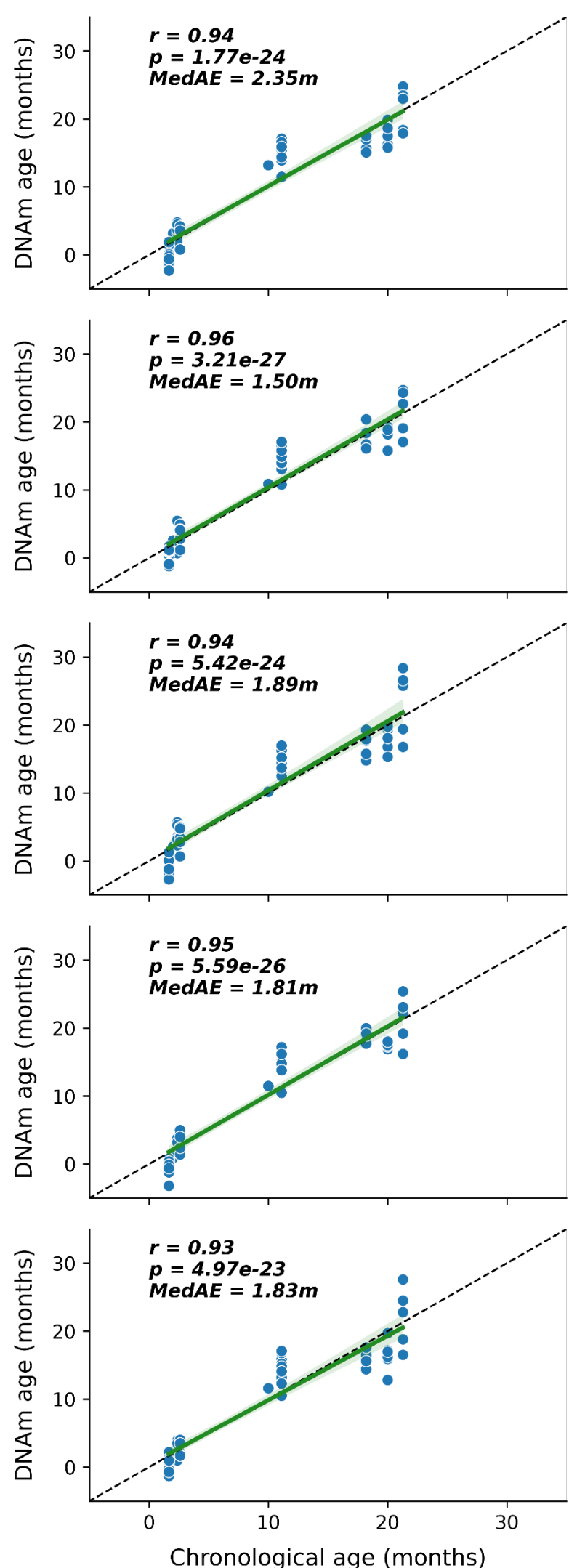

Chronological age (months)

\section{Petkovich et al. models}
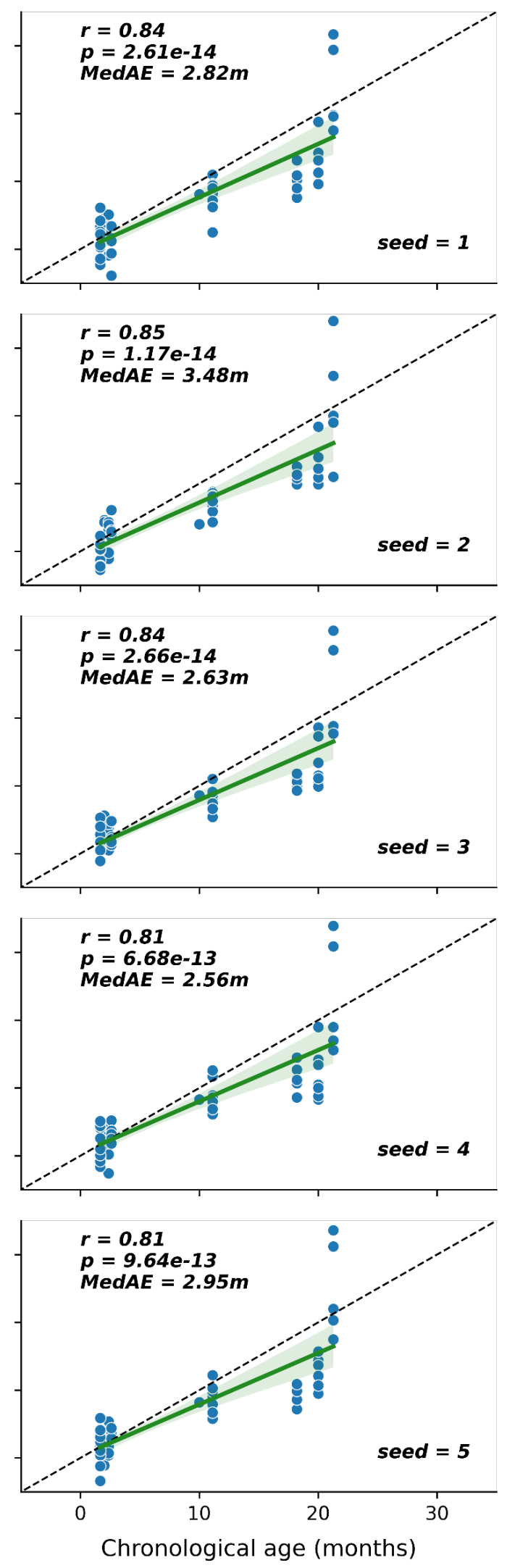


\section{Extended Data Figure 1: Low-pass epigenetic age predictions in the Thompson et al. blood dataset}

Scatterplots of epigenetic age predictions for C57BL/6J blood samples in the Thompson et al. dataset $(\mathrm{n}=50)$. Panels on the left depict predictions using Thompson et al. reference models, while panels on the right show predictions using regression models computed with Petkovich et al. data. The particular random downsampling seed used is shown in the bottom right and corresponds across training datasets. The Pearson correlation coefficient $(r)$, the associated twotailed p-value $(p)$, and the median absolute error $(M e d A E)$ is shown for each plot. Data shown are from the best performing set of parameters $(100,000$ reads and 1,000 CpGs included in the likelihood profile), based on benchmarking (see Figure 2). Regression lines (green) are shown with $95 \%$ confidence intervals (light green). The dashed lines represent the identity between epigenetic (DNAm) and chronological age. 


\section{Petkovich et al. dataset}

$n=153$

Thompson et al. models
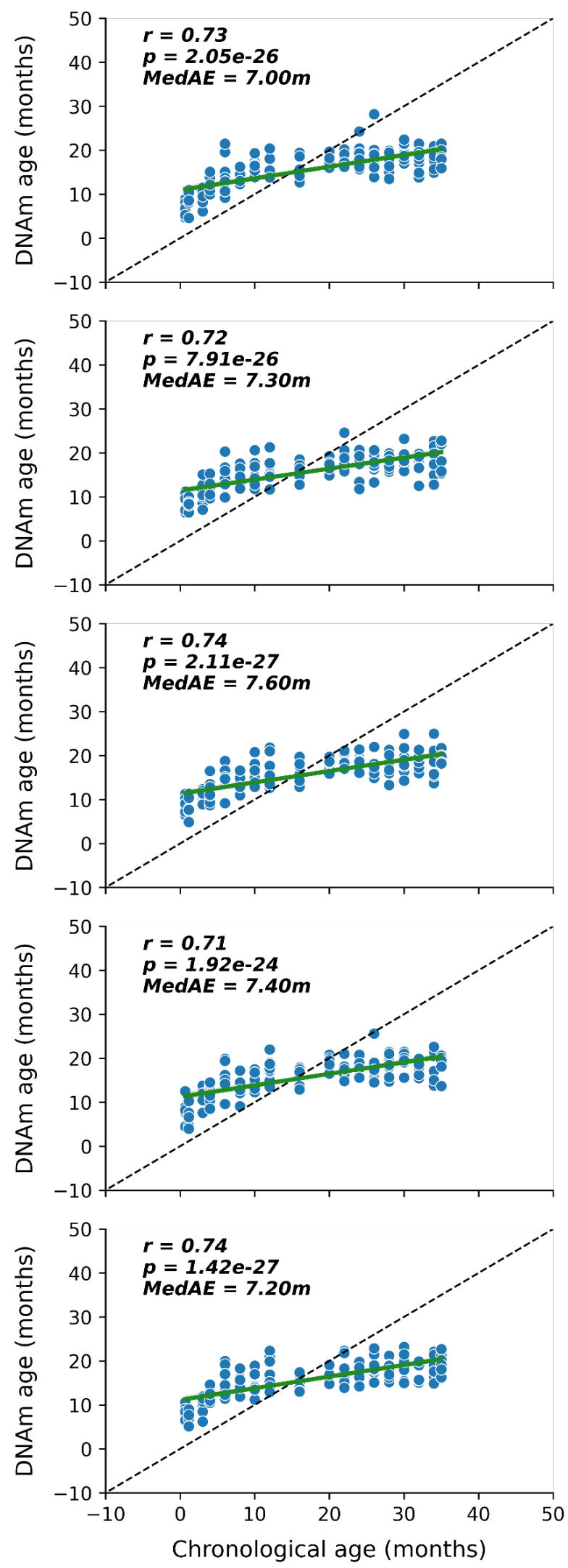

\section{Petkovich et al. models}
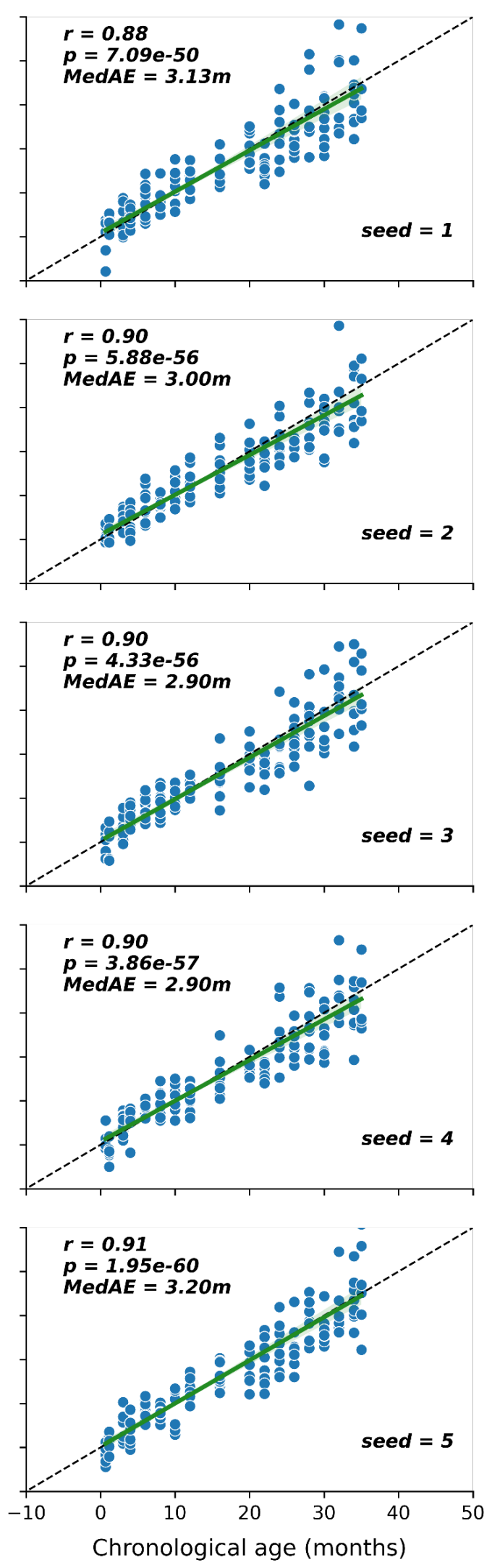


\section{Extended Data Figure 2: Low-pass epigenetic age predictions in the Petkovich et al. blood dataset}

Scatterplots of epigenetic age predictions for C57BL/6J blood samples in the Petkovich et al. dataset $(\mathrm{n}=153)$. Panels on the left depict predictions using Thompson et al. reference models, while panels on the right show predictions using Petkovich et al. regression models. The particular random downsampling seed used is shown in the bottom right and corresponds across training datasets. The Pearson correlation coefficient $(r)$, the associated two-tailed $\mathrm{p}$-value $(p)$, and the median absolute error $(M e d A E)$ is shown for each plot. Data shown are from the best performing set of parameters (100,000 reads and 500 top CpGs included in the likelihood profile), based on benchmarking (see Figure 3). Regression lines (green) are shown with 95\% confidence intervals (light green). The dashed lines represent the identity between epigenetic (DNAm) and chronological age. 
a

b
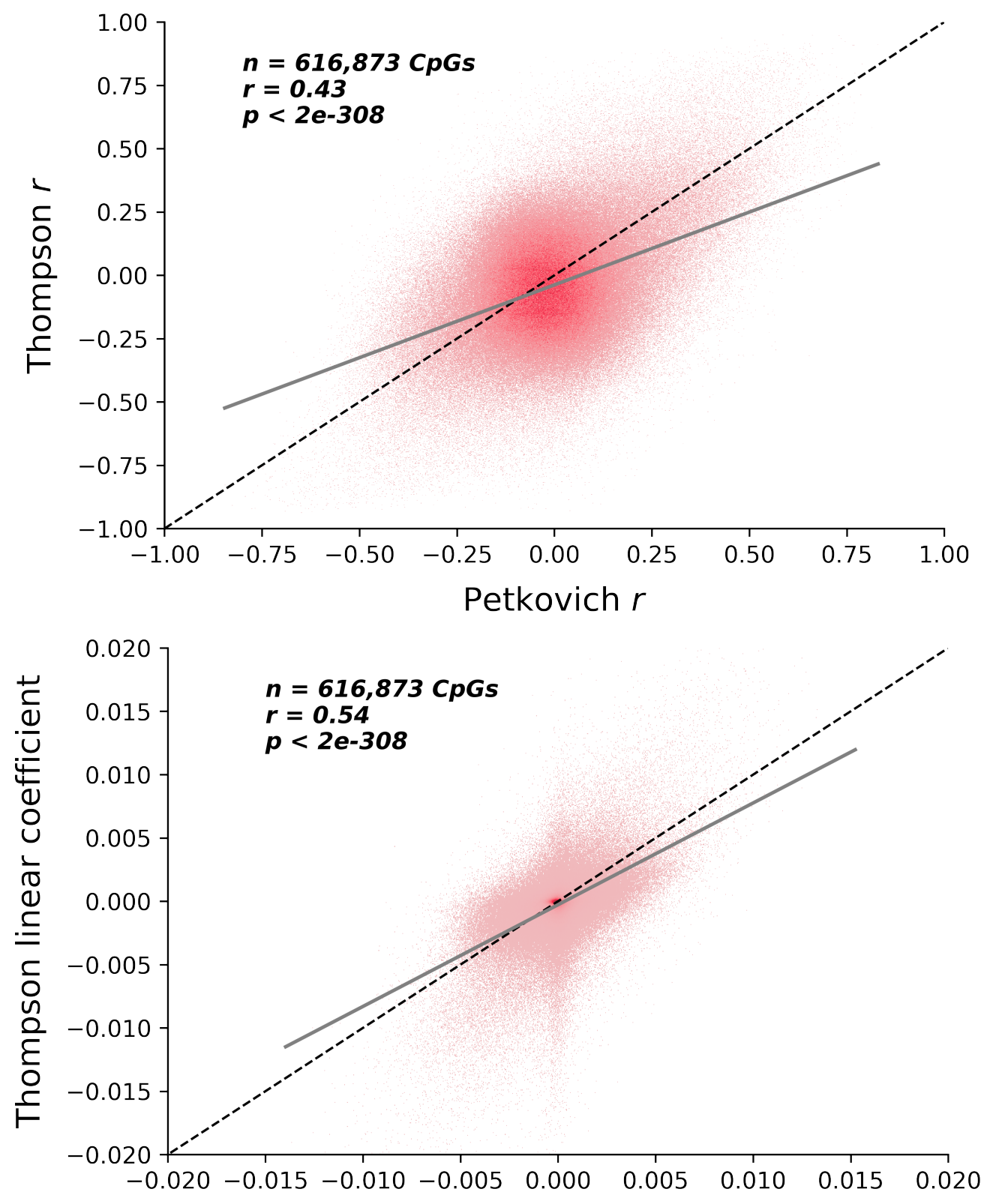

C

Petkovich linear coefficient

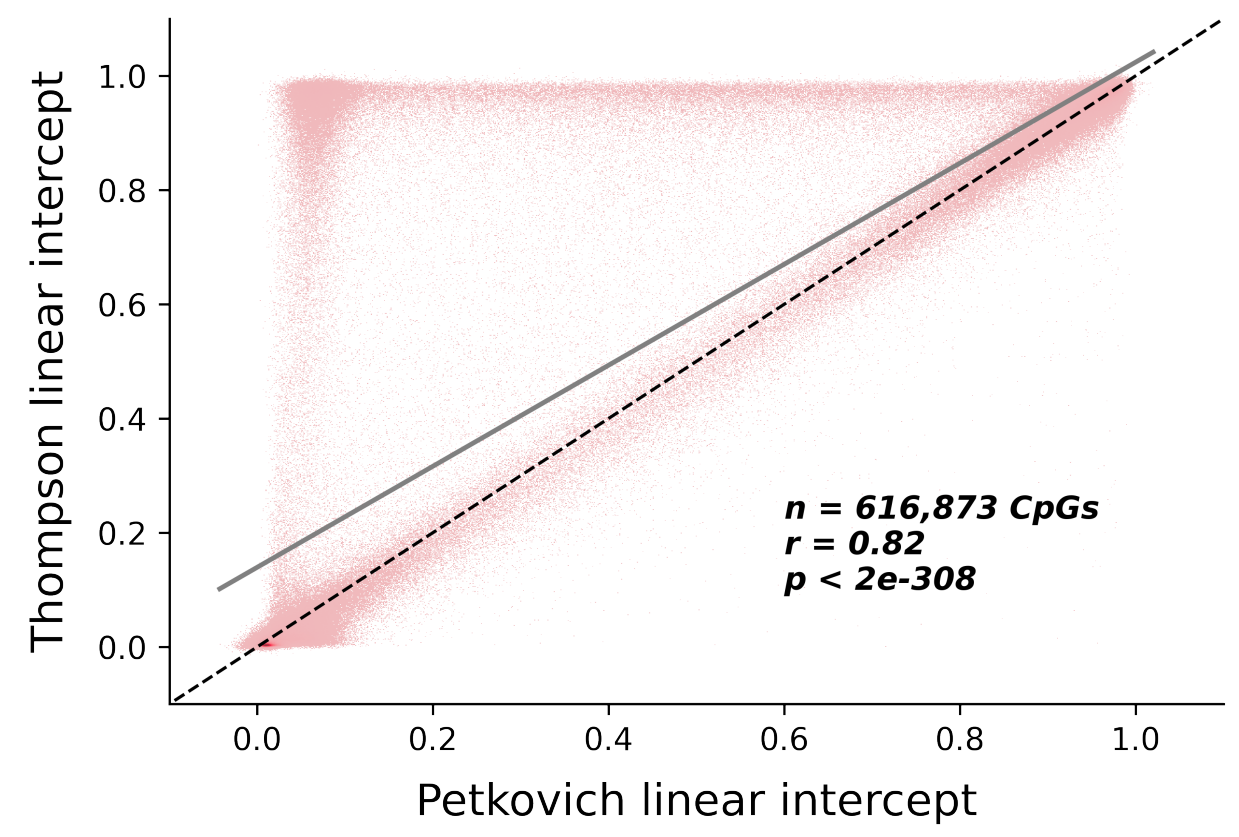




\section{Extended Data Figure 3: Comparison of linear association metrics with age in the Thompson et al. and Petkovich et al. datasets}

Density plots depicting the correspondence of (a) Pearson correlation coefficients, (b) linear regression coefficients, and (c) linear regression intercepts between training data from Petkovich

et al. (x-axis) and Thompson et al. (y-axis). The number of common CpGs between both datasets is shown $(n)$, as well as the Pearson correlation coefficient $(r)$ and the associated two-tailed pvalue. Dashed lines represent identity between a metric in the Petkovich et al. dataset and that same metric in the Thompson et al. dataset. Regression lines (grey) are shown with 95\% confidence intervals (light grey). 


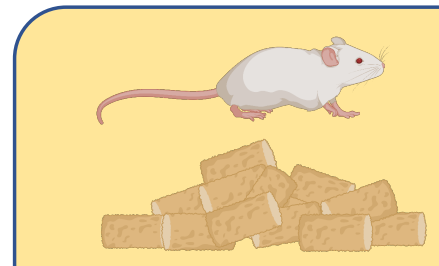

Ad libitum

\section{Petkovich et al. dataset

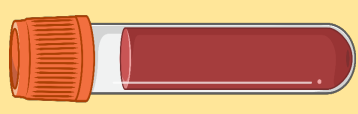 \\ Caloric restriction \\ Con}

Thompson et al. models
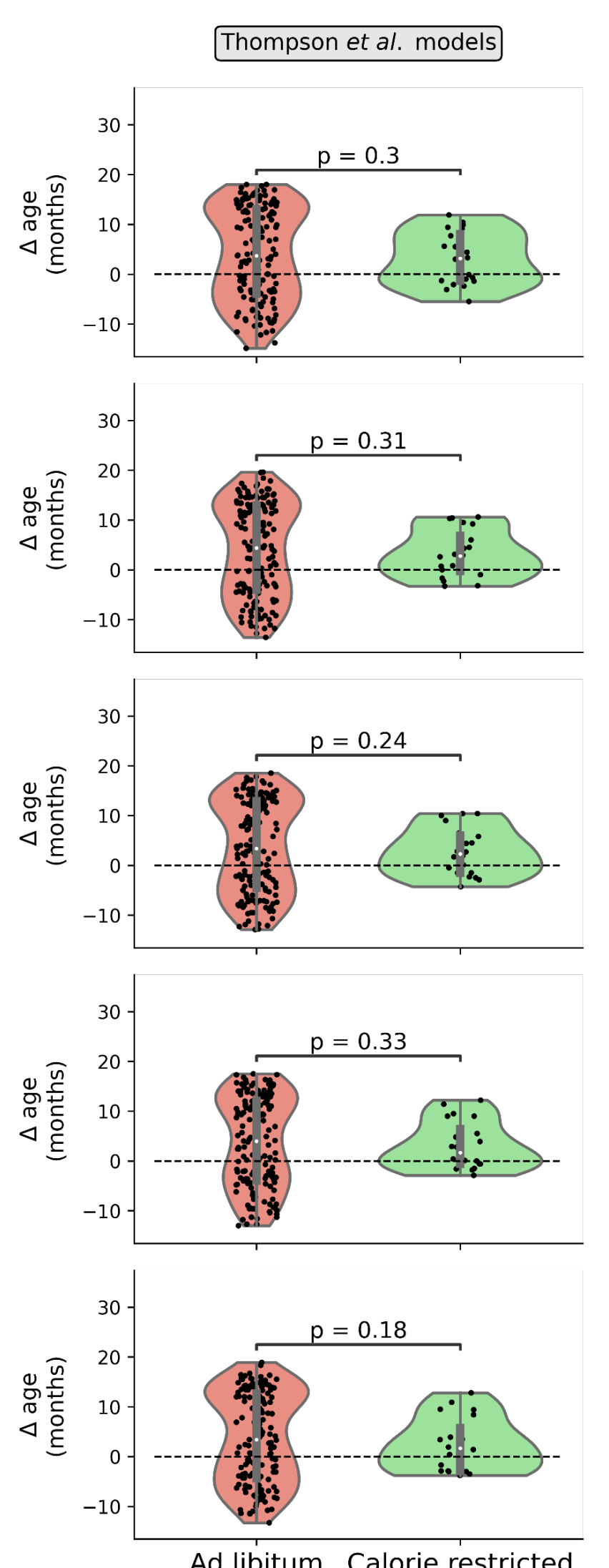

Petkovich et al. models
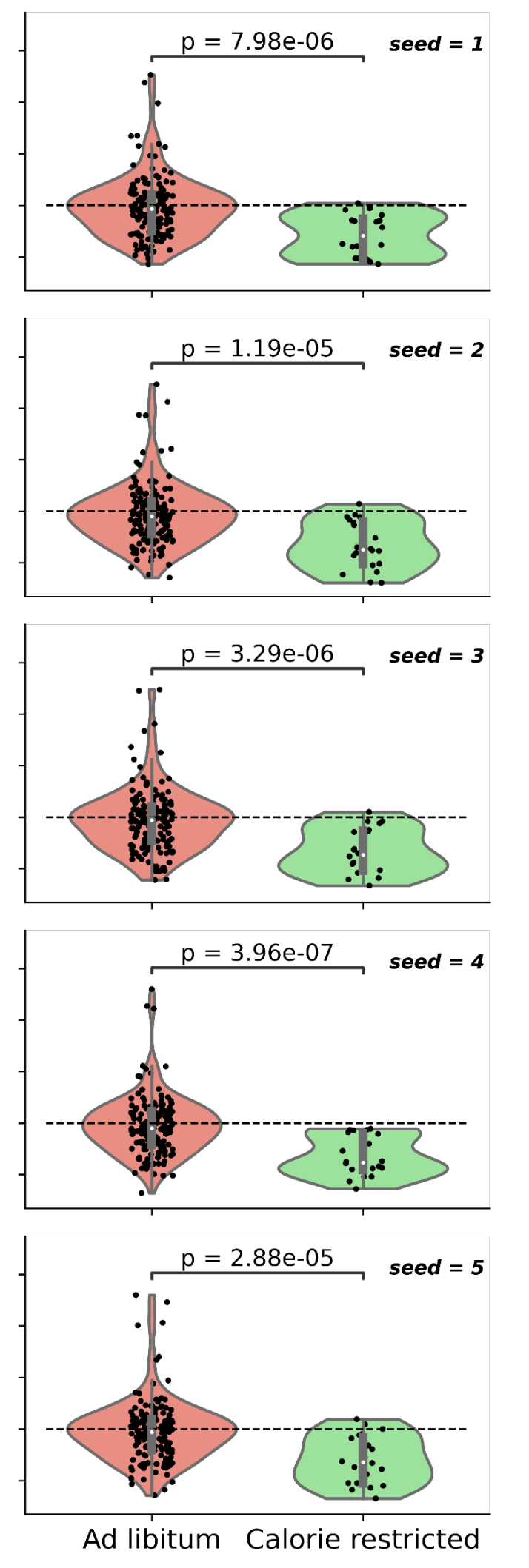

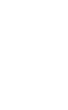

Ad libitum Calorie restricted

)




\section{Extended Data Figure 4: Decreased delta age in calorically-restricted samples}

Violin plots of delta age (epigenetic age - chronological age) for ad libitum $(\mathrm{n}=153$, red) and calorically-restricted $(\mathrm{n}=20$, green) male C57BL/6J blood samples in the Petkovich et al. dataset. Panels on the left depict predictions using Thompson et al. reference models, while panels on the right show predictions leveraging Petkovich et al. data. The particular random downsampling seed used is shown in the top right. The p-values depicted are derived from Welch's one-tailed t-test (assuming unequal variances). Data shown are from the best performing set of parameters (100,000 reads per sample and 2,500 CpGs in the likelihood profile) based on benchmarking. Inner boxplots depict the median and the $1^{\text {st }}$ and $3^{\text {rd }}$ quartiles, with whiskers extending to $1.5 \mathrm{x}$ the interquartile range. Violin plots show the kernel density estimation of the data. Dashed lines highlight a delta age of 0 . 

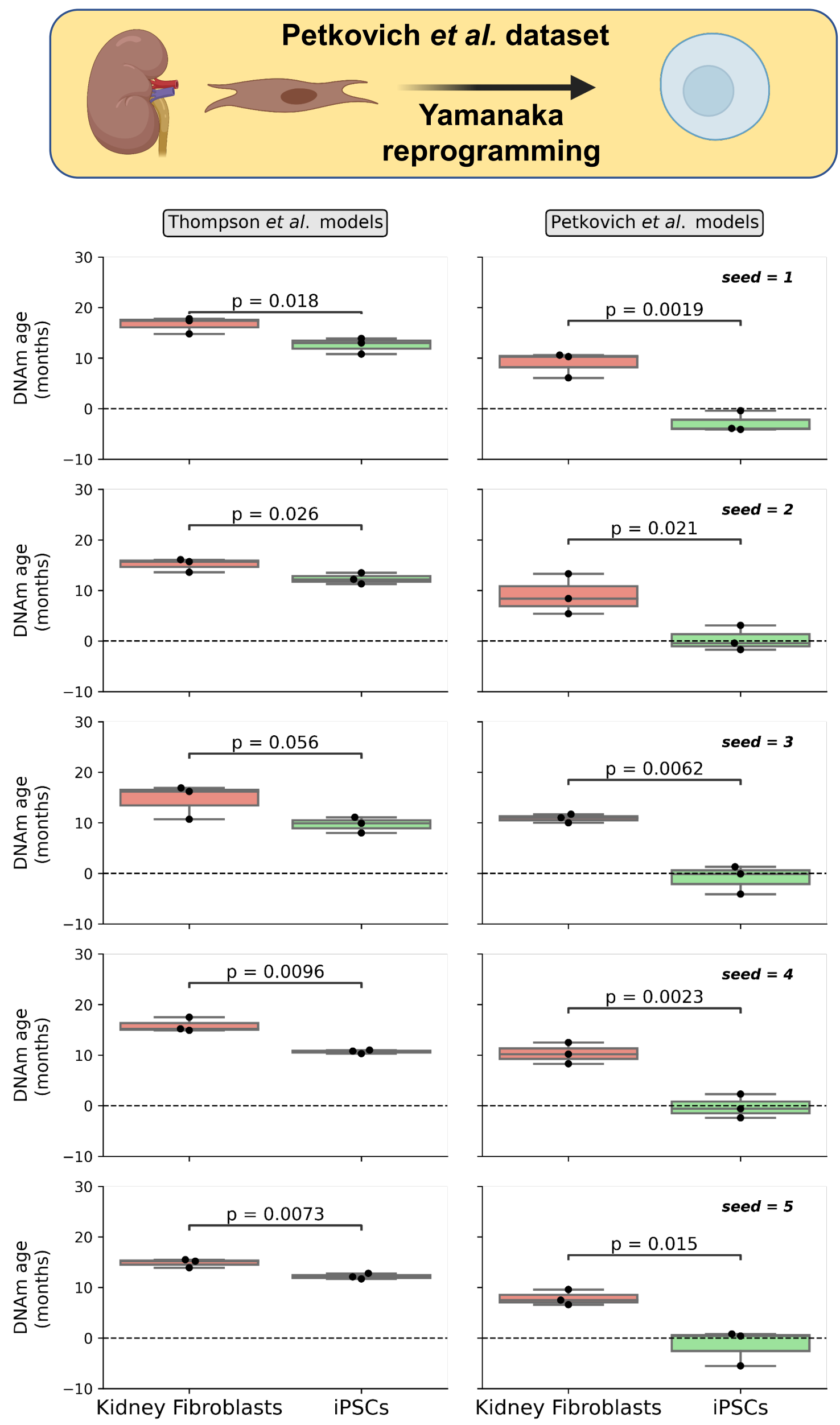


\section{Extended Data Figure 5: Age reversal assessed by low-pass scAge in renal fibroblasts and derived iPSCs}

Box plots of epigenetic age for kidney fibroblasts $(\mathrm{n}=3$, red $)$ and kidney-derived induced pluripotent stem cells (iPSCs, $\mathrm{n}=3$, green) from C57BL/6J mice in the Petkovich et al. ${ }^{10}$ dataset. Panels on the left depict predictions using Thompson et al. reference models, while panels on the right show predictions using Petkovich et al. regression models. The particular random downsampling seed used is shown in the top right and corresponds across training datasets. The $\mathrm{p}$-values depicted are derived from Welch's one-tailed t-test (assuming unequal variances). Data shown are from the best performing set of parameters $(100,000$ reads per sample and the top 500 age-associated CpGs in the likelihood profile). Boxplots depict the median and the $1^{\text {st }}$ and $3^{\text {rd }}$ quartiles, with whiskers extending to $1.5 \mathrm{x}$ the interquartile range. Dashed lines indicate an epigenetic (DNAm) age of 0. 

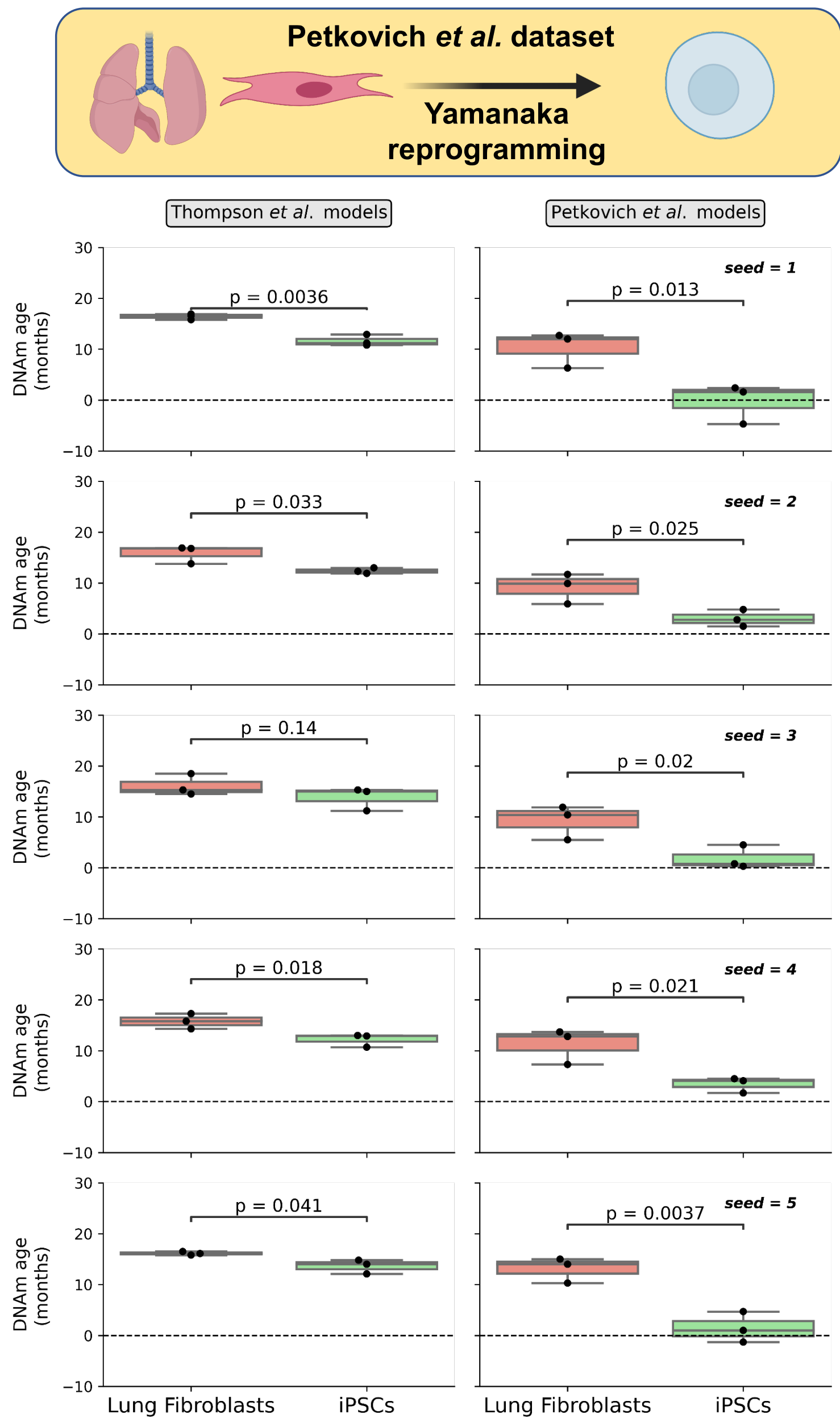


\section{Extended Data Figure 6: Age reversal assessed by low-pass scAge in lung fibroblasts and derived iPSCs}

Box plots of epigenetic age for lung fibroblasts $(n=3$, red) and lung-derived induced pluripotent stem cells (iPSCs, $\mathrm{n}=3$, green) from C57BL/6J mice in the Petkovich et al. ${ }^{10}$ dataset. Panels on the left depict predictions using the Thompson et al. training dataset, while panels on the right show predictions using the Petkovich et al. regression models. The particular random seed used is shown in the top right and corresponds across training datasets. The p-values depicted are derived from Welch's one-tailed t-test (assuming unequal variances). Data shown are from the best performing set of parameters $(100,000$ reads per sample and the top 500 age-associated CpGs in the likelihood profile). Boxplots depict the median and the $1^{\text {st }}$ and $3^{\text {rd }}$ quartiles, with whiskers extending to $1.5 \mathrm{x}$ the interquartile range. Dashed lines indicate an epigenetic (DNAm) age of 0 . 


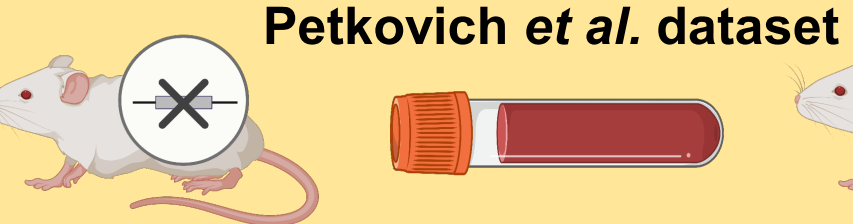

\section{GHRKO}

\section{Control}

Thompson et al. models
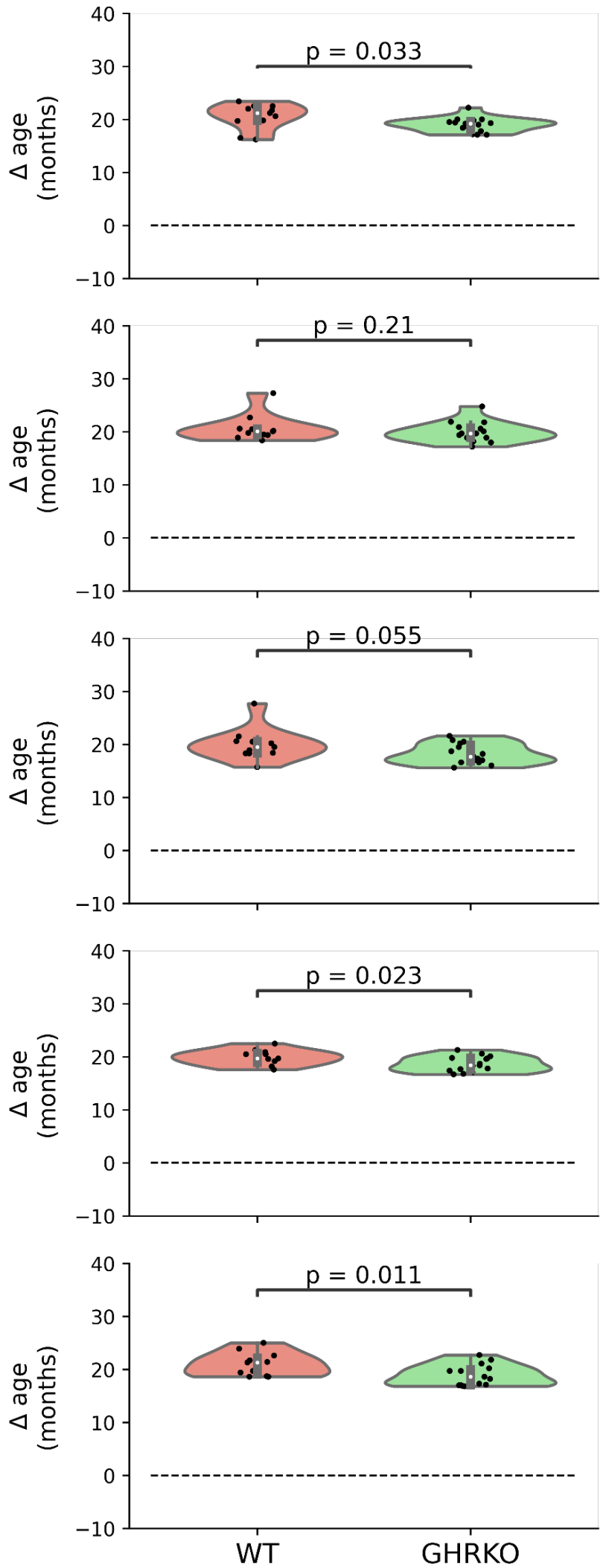

Petkovich et al. models
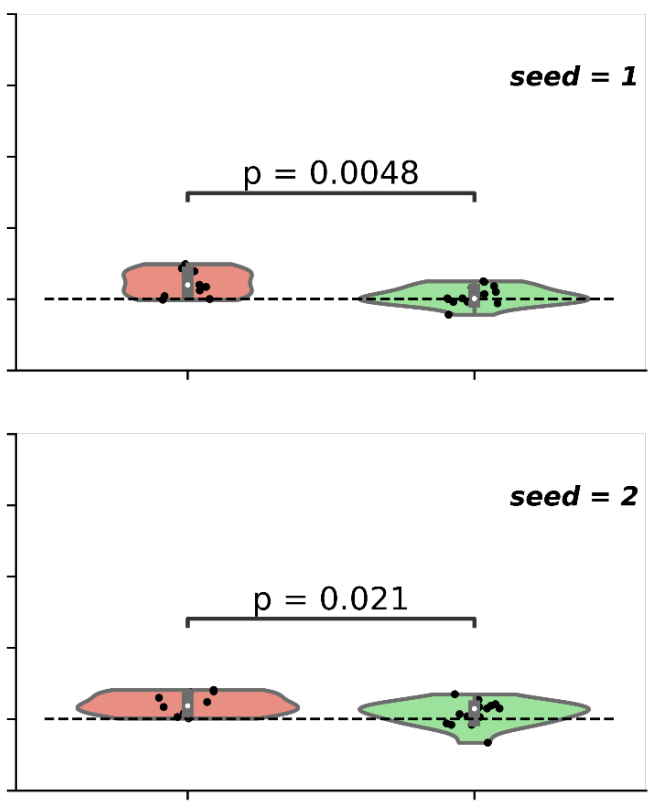

seed $=3$
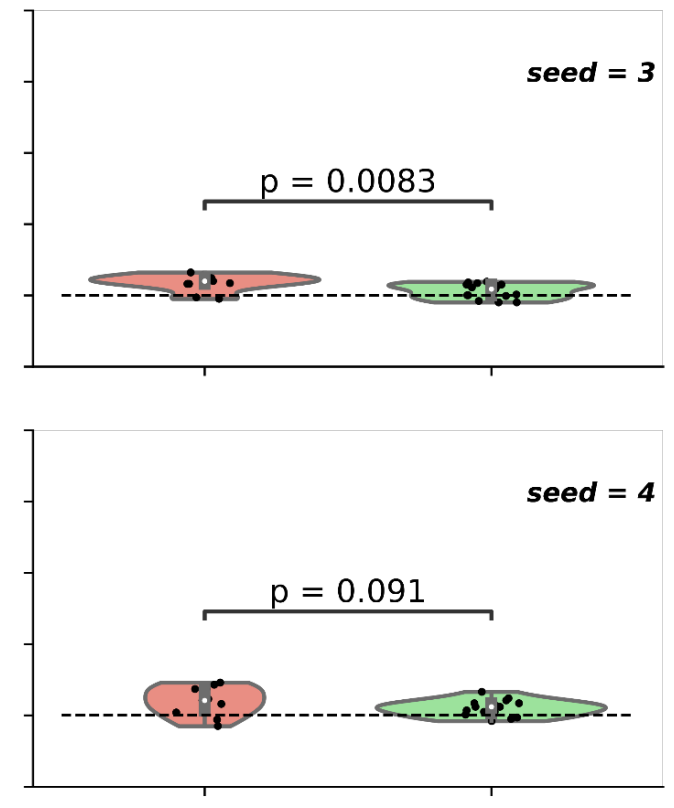

seed $=5$

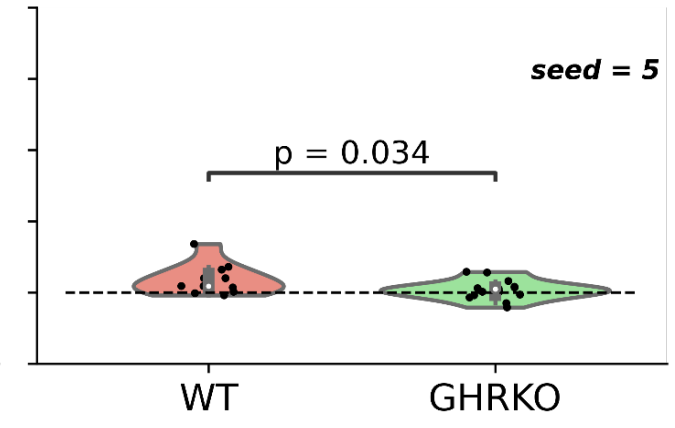




\section{Extended Data Figure 7: Decreased delta age in GHRKO samples}

Violin plots of delta age (epigenetic age - chronological age) for wild type $(\mathrm{n}=11$, red) and growth hormone receptor knockout (GHRKO, $\mathrm{n}=15$, green) samples from (C57BL/6J $\mathrm{x}$ $\mathrm{BALB} / \mathrm{cByJ}) / \mathrm{F} 2$ mice in the Petkovich et al. ${ }^{10}$ dataset. Panels on the left depict predictions using Thompson et al. reference models, while panels on the right show predictions using Petkovich et al. regression models. The particular random downsampling seed used is shown in the top right and corresponds across training datasets. The p-value depicted is derived from Welch's onetailed t-test (assuming unequal variances). Data shown are from the best performing set of parameters (100,000 reads per sample with the top 10,000 CpGs included in the scAge profile). Inner boxplots depict the median and the $1^{\text {st }}$ and $3^{\text {rd }}$ quartiles, with whiskers extending to $1.5 \mathrm{x}$ the interquartile range. Violin plots show the kernel density estimation of the data. Dashed lines indicate a delta age of 0 . 


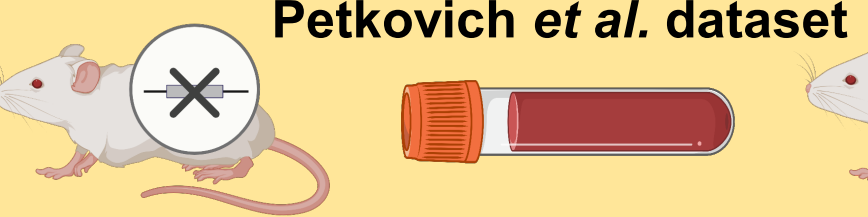

Snell dwarf

Thompson et al. models
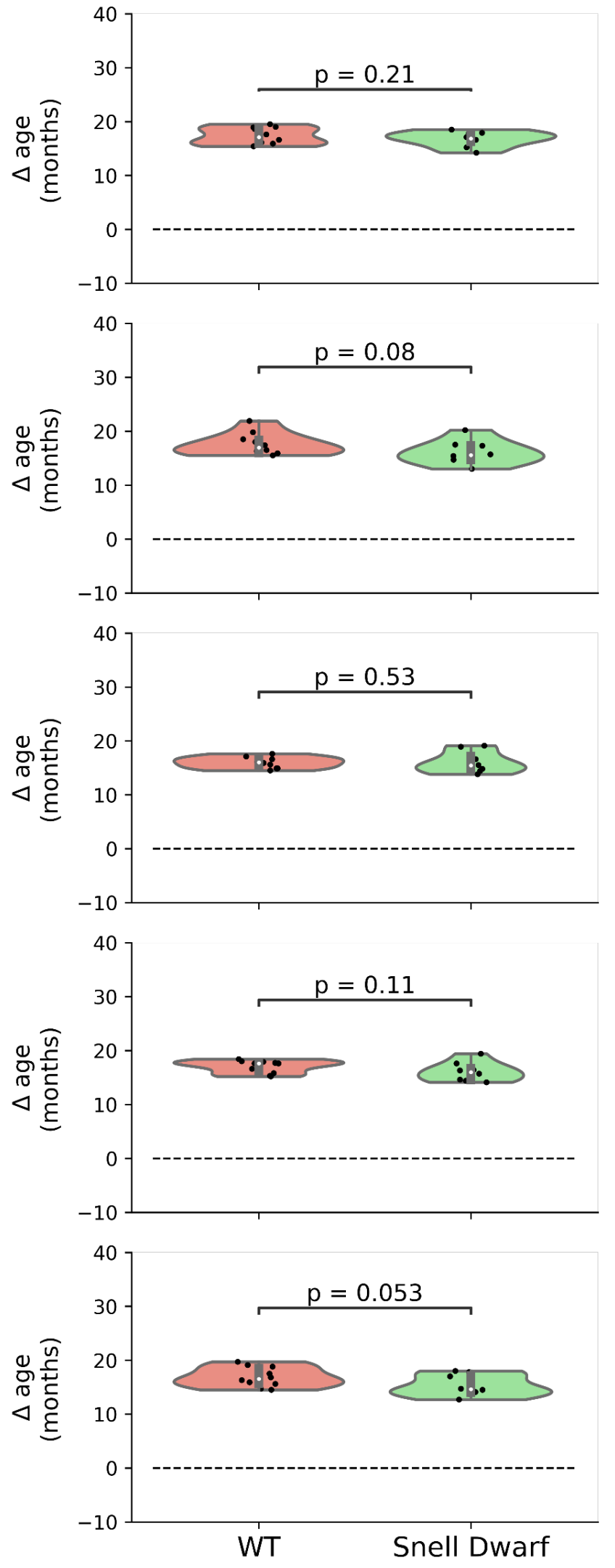

Petkovich et al. models
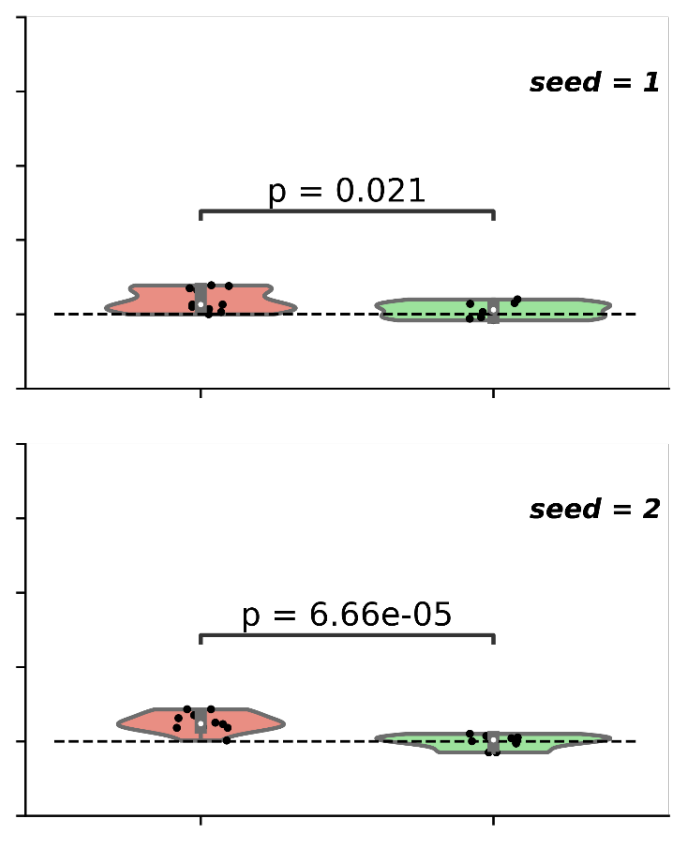

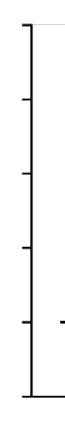

$$
\text { seed }=3
$$
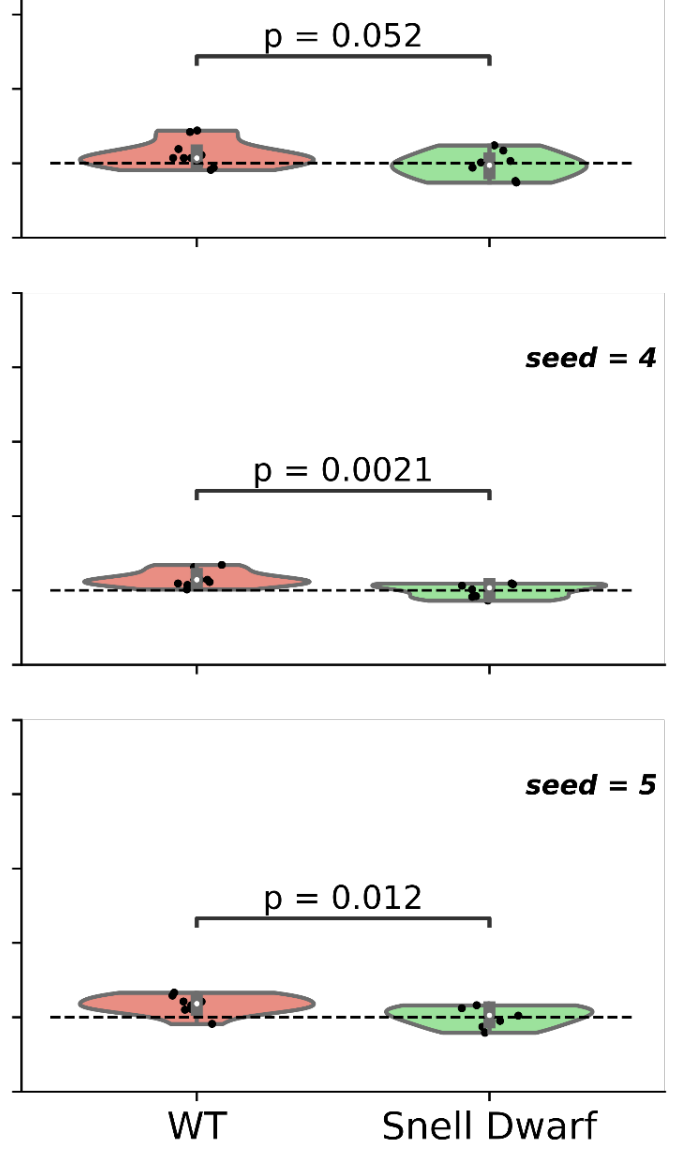


\section{Extended Data Figure 8: Decreased delta age in Snell dwarf samples}

Violin plots of delta age (epigenetic age - chronological age) for wild type $(\mathrm{n}=10$, red) and Snell Dwarf $\left(\mathrm{n}=8\right.$, green) samples from $(\mathrm{DW} / \mathrm{J} \times \mathrm{C} 3 \mathrm{H} / \mathrm{HEJ}) / \mathrm{F} 2$ mice in the Petkovich et al. $^{10}$ dataset. Panels on the left depict predictions using Thompson et al. reference models, while panels on the right show predictions using Petkovich et al. models. The particular random seed used is shown in the top right and corresponds across training datasets. The p-value depicted is derived from Welch's one-tailed t-test (assuming unequal variances). Data shown are from the best performing set of parameters (100,000 reads per sample with the top 5,000 CpGs included in the profile). Inner boxplots depict the median and the $1^{\text {st }}$ and $3^{\text {rd }}$ quartiles, with whiskers extending to $1.5 \mathrm{x}$ the interquartile range. Violin plots show the kernel density estimation of the data. Dashed lines indicate a delta age of 0 . 